\title{
Revision and significance of the Westphalian (Middle Pennsylvanian) arborescent lycopsid Bergeria dilatata (Lindley \& Hutton) Álvarez-Vázquez \& Wagner
}

\author{
Carmen ÁLVAREZ-VÁZQUEZ ${ }^{*}$, Jiři BEK ${ }^{2}$, John A. KNIGHT \& Robert H. WAGNER ${ }^{l}$
}

\author{
${ }^{1}$ Centro Paleobotánico, Real Jardín Botánico de Córdoba, Avenida de Linneo, s/n, 14004 Córdoba, Spain; paleo.calvarez@ \\ jardinbotanicodecordoba.com; jaknightuk@btinternet.uk \\ ${ }^{2}$ Laboratory of Palaeobiology and Palaeoecology, Institute of Geology, Academy of Sciences of the Czech Republic v.v.i., \\ Rozvojová 269, 16500 Prague 6, Czech Republic; bek@gli.cas.cz \\ * Corresponding author
}

Álvarez-Vázquez, C., Bek, J., Knight, J.A. \& Wagner, R.H. 2018. Revision and significance of the Westphalian (Middle Pennsylvanian) arborescent lycopsid Bergeria dilatata (Lindley \& Hutton) Álvarez-Vázquez \& Wagner. [Revisión e importancia de la lycópsida arborescente del Westfaliense (Pensilvánico Medio) Bergeria dilatata (Lindley \& Hutton) Álvarez-Vázquez \& Wagner]. Spanish Journal of Palaeontology, 33 (1), 5-40.

\begin{abstract}
Bergeria dilatata (Lindley \& Hutton) Álvarez-Vázquez \& Wagner, a Middle Pennsylvanian lycopsid, is redescribed based on type material from England as well as 586 specimens from the Peñarroya-Belmez-Espiel Coalfield, Córdoba province, southern Spain. The species is characterized by longer than broad, smooth leaf cushions showing false leaf scars, and the presence of perennial, long, entire, single-veined leaves. A complete synonymy is presented and 21 new species (two of them with doubts), previously placed in 6 different genera, have been synonymized. Associated Flemingites-type cones, determined as Flemingites russelianus, yielded in situ spores of the Lagenoisporites rugosus type and microspores of the Microspinosporites type.
\end{abstract}

Keywords: Bergeria dilatata, Flemingites russelianus, Lycopsida, Westphalian, Peñarroya-Belmez-Espiel Coalfield.

\section{RESUMEN}

Bergelia dilatata (Lindley \& Hutton) Álvarez-Vázquez \& Wagner, una licópsida del Pensilvánico medio, es redescrita en base al material tipo inglés y a 586 ejemplares procedentes de la cuenca carbonífera de Peñarroya-Belmez-Espiel, provincia de Córdoba, sur de España. La especie se caracteriza por tener cojinetes foliares más largos que anchos, no ornamentados, con falsas cicatrices foliares, y por la presencia de hojas perennes, largas, enteras, univervias. Se presenta una sinonimia completa, incluyéndose en la misma 21 nuevas especies (dos de ellas con dudas) anteriormente atribuídas a 6 géneros diferentes. Los estróbilos asociados, de tipo Flemingites, han sido determinados como Flemingites russelianus, y contienen esporas in situ de tipo Lagenoisporites rugosus y microsporas de tipo Microspinosporites.

Palabras clave: Bergeria dilatata, Flemingites russelianus, Lycopsida, Westfaliense, cuenca minera Peñarroya-BelmezEspiel. 


\section{INTRODUCTION}

The classic Lepidodendron Sternberg, 1820 is perhaps the most frequently quoted form-genus of Carboniferous lycopsids (see Fossilium Catalogus Plantae - Jongmans, 1913, 1929, 1936; Jongmans \& Dijkstra, 1969; Dijkstra \& van Amerom, 1991, 1994). It is characterised by spirally arranged, vertically elongated, rhomboidal to fusiform leaf cushions that are either contiguous or separated by narrow grooves; a rhomboidal to subtriangular leaf scar is normally situated near the middle of the cushion or a little above. The leaf scar exhibits the presence of three small pit-like impressions, the central one representing the vascular trace, and the two lateral ones aerating tissue (parichnos). In Lepidodendron sensu stricto two infrafoliar parichnos markings occur below the leaf scar. The presence of leaf scar and infrafoliar parichnos are determinative characters at the generic level. Although known (see references in Álvarez-Vázquez \& Wagner, 2014, p. 172), stem remains with attached leaves are not normally preserved.

Lepidodendron, being a classic genus, has often been laxly interpreted. In fact, we consider that only a few of the more than 400 species recorded as Lepidodendron actually belong to this genus. Several different genera have been taken out of Lepidodendron, mainly (but not exclusively) on the basis of anatomical differences. Examples are: Paralycopodites (Morey \& Morey, 1977), Diaphorodendron (DiMichele, 1985), Hizemodendron (Bateman \& DiMichele, 1991) and Synchysidendron (DiMichele \& Bateman, 1992, 1993).

In addition, two species initially attributed to Lepidodendron were transferred by Álvarez-Vázquez \& Wagner (2014) to the little known form-genus Bergeria Presl in Sternberg, 1838: these are Bergeria dilatata (Lindley \& Hutton) Álvarez-Vázquez \& Wagner and Bergeria worthenii (Lesquereux) Álvarez-Vázquez \& Wagner. The former is reviewed in the present paper, based in the first place on the type specimen (Lepidodendron dilatatum Lindley \& Hutton, 1831, pl. 7, fig. 2) from northern England. Secondly, a large collection from the Peñarroya-Belmez-Espiel Coalfield, in SW Spain, forms the basis for characterizing the range of variation recorded herein.

\section{MATERIAL}

More than 600 specimens (branches, leafy twigs and associated strobili), collected from upper Langsettian/ lower Duckmantian and lower Bolsovian strata of the Peñarroya-Belmez-Espiel Coalfield (Fig. 1), have been available for examination. Specimens are preserved as adpressions and were obtained, firstly, from boreholes and exploratory trenches made in the 1970s as part of an exploration programme by the Empresa Nacional Adaro de Investigaciones Mineras (ENADIMSA). Further material was obtained later, in the 1980s and 1990s, as a result of systematic exploration by the Geology Department of the Empresa Nacional Carbonífera del Sur, S.A. (ENCASUR) (mainly boreholes). In the years between 2003 and 2012 this collection has increased substantially by sustained sampling in the La Ballesta opencast site, at c. $5 \mathrm{~km}$ ESE of the village of Espiel (Fig. 1). More than half of the available specimens have come from this opencast mine (including exploratory boreholes). The large number of specimens from Peñarroya now allows morphological variation to be characterized.

The material from Spain is compared with the type material of Lindley \& Hutton's Lepidodendron dilatatum and Lepidodendron gracile, both of which we regard as synonymous. The holotypes of these species are here figured photographically for the first time (Figs 2 and 3, respectively). These specimens are curated as part of the Hutton Collection, housed in the Great North MuseumHancock, Newcastle-upon-Tyne, England.

In the collections from the Peñarroya-Belmez-Espiel Coalfield some 23 specimens of characteristic strobili have been found in close association with stems and leafy twigs of Bergeria dilatata. Whereas direct connection has not been unequivocally demonstrated, these are the only lycopsid fertile elements in assemblages dominated by Bergeria dilatata. The strobili are elongate and bisporangiate and morphologically conform to the genus Flemingites Carruthers emend. Brack-Hanes \& Thomas. Two of these strobili have been submitted to palynological investigation in the laboratories of the Institute of Geology, Prague, Czech Republic, which also undertook bulk maceration of associated sediment from the same collection horizons. In situ megaspores have been identified as Lagenoisporites rugosus type and in situ microspores as of Microspinosporites orbiculus type. These identifications are entirely consistent with in situ megaspores and microspores reported in strobili of Flemingites (Bek, 2013, 2017). The morphological characteristics of the strobili conform to Flemingites russelianus (Binney) Brack-Hanes \& Thomas, as presented below.

\section{GEOLOGICAL SETTING}

The Peñarroya-Belmez-Espiel Coalfield occurs in a narrow NW-SE striking strip in the northwestern part of the province of Córdoba, SW Spain (Fig. 1), formed within a strike-slip controlled basin. Its preserved width nowhere exceeds $3 \mathrm{~km}$ and the current outcrop length is some $50 \mathrm{~km}$, 


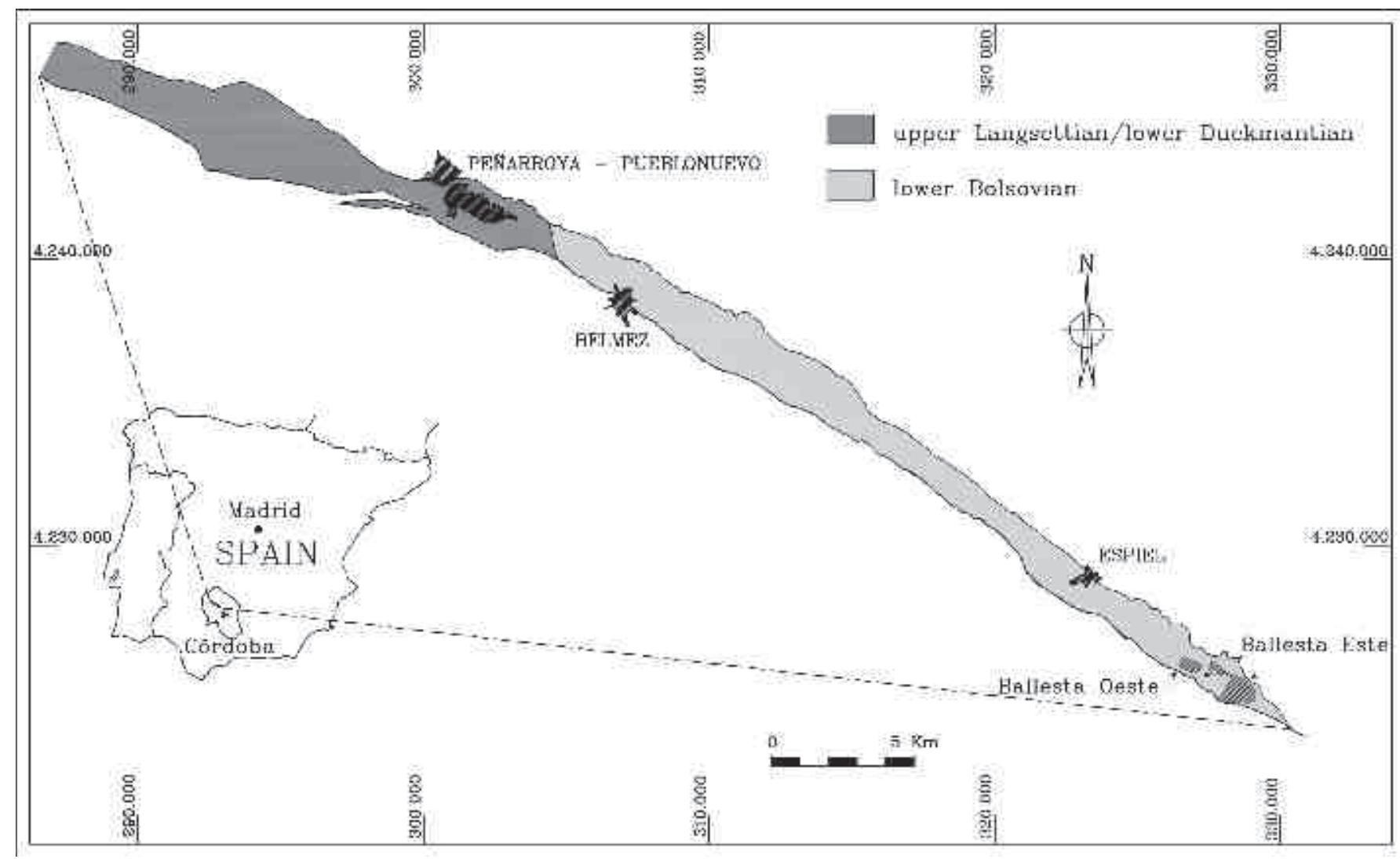

Figure 1. Sketch map of the Peñarroya-Belmez-Espiel Coalfield showing the position of the two sub-basins and location of the Ballesta opencast site. Simplified from Álvarez-Vázquez \& Cleal (2016).

although the depositional extent is estimated to have been considerably greater. The outcrop is concealed by Tertiary strata at its north-western extreme and to the south-east the outcrop wedges out against the strike-slip fault zone on the southern border of the coalfield. The coalfield has been described (Wagner, 1999, 2004, 2013) as controlled by a strike-slip fault comparable to the present-day San Andreas Fault in California, with a deduced lateral displacement of c. $150 \mathrm{~km}$ (Wagner, 2103).

Sub-basins have been dated as late Langsettian/early Duckmantian and (late Duckmantian?) early Bolsovian based on megafloral associations (Álvarez-Vázquez, 1995, 1999; Wagner \& Álvarez-Vázquez, 2010). A full documentation of these well-sampled floras is still an ongoing process, but so far 130 taxa have been found in the two consecutive sub-basins. The total flora is based on the analysis of c. 22,000 samples with a total of some 50,000 plant fragments. This is the most complete record of a lower to middle Westphalian megaflora in the Iberian Peninsula.

The Peñarroya flora is broadly comparable with that of the well-studied Paralic Coal Belt of northern Europe, from South Wales through central and southern England into northern France, Belgium and the Ruhr District in western Germany. The fern, lycopsid and sphenopsid species contents of the Peñarroya flora are exactly the same as in northern Europe. However, pteridosperm taxa are fewer; and, for the time being, the newly described Wagneropteris minima (Álvarez-Vázquez \& Cleal, $2016)$ is the only endemic species. With regard to the Lycopsida, 19 stem taxa have been determined: Bergeria dilatata (the one described here), Bergeria worthenii, Lepidodendron aculeatum Sternberg, Lepidodendron bellii Álvarez-Vázquez \& Wagner, "Lepidodendron" aff. lossenii Weiss, Lepidophloios laricinus (Sternberg) Sternberg, Diaphorodendron decurtatum (Dawson) Álvarez-Vázquez \& Wagner, Bothrodendron minutifolium (Boulay) Zeiller, cf. Eleutherophyllum waldenburgense (Stur) Zimmermann, Selaginella sp., a new species of Polysporia (ÁlvarezVázquez et al., in prep.), Asolanus camptotaenia Wood, Sigillaria boblayi Brongniart, Sigillaria davreuxii Brongniart, Sigillaria elegans Brongniart, Sigillaria ovata Sauveur, Sigillaria polyploca Boulay, Sigillaria reticulata Lesquereux, and several specimens of a still indeterminate stem with ulodendroid scars.

The above taxa are represented together by nearly 500 specimens, significantly less than the number of specimens of Bergeria dilatata studied here, and only about $2 \%$ of the total megaflora of the basin. 


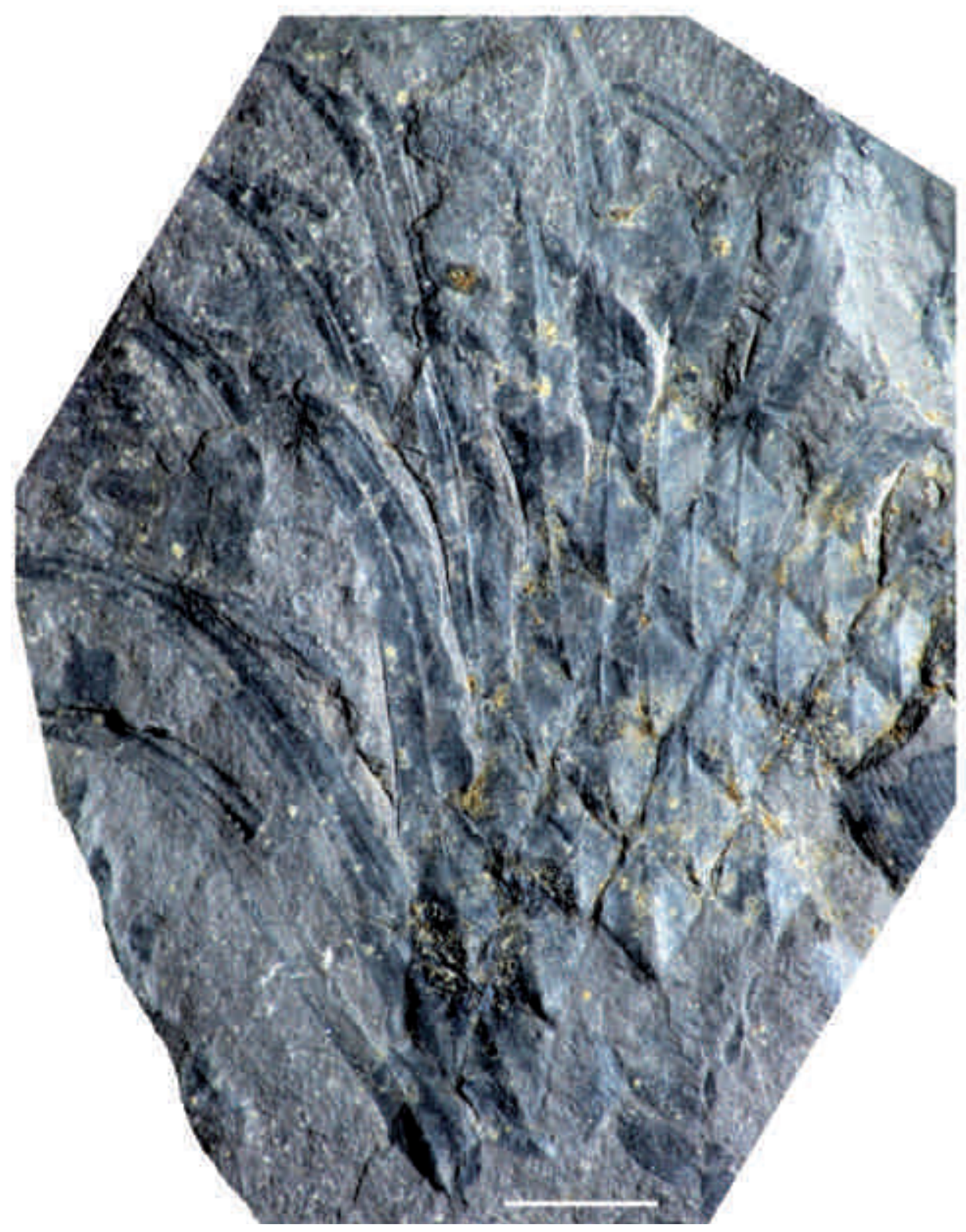

Figure 2. Bergeria dilatata (Lindley \& Hutton) Álvarez-Vázquez \& Wagner. HM G02.16 (x 2). Holotype of Lepidodendron dilatatum. Figured as a drawing by Lindley \& Hutton (1831, pl. 7, fig. 2). Stem fragment showing smooth, rhomboidal cushions and the characteristic long, entire, single-veined leaves inserted in the uppermost part of leaf cushions. Note the close resemblance with the stem fragment with attached leaves figured as Lepidodendron ophiurus by Zeiller (1886, pl. LXVIII, fig. 4); also the shape of leaf cushions with those of Presl's illustrations of Bergeria angulata, Bergeria quadrata and Bergeria rhombica. Origin: Low Main coal seam, Felling Colliery, near Newcastle-upon-Tyne; lower Duckmantian. Scale bar $=1 \mathrm{~cm}$. Repository: Great North Museum-Hancock, Newcastle-upon-Tyne, England.

\section{SYSTEMATIC PALAEONTOLOGY}

\section{Class LYCOPSIDA Scott, 1909}

\section{Order LEPIDODENDRALES}

Family Flemingitaceae Thomas \& Brack-Hanes, 1984

Genus Bergeria Presl in Sternberg, 1838

Type species Bergeria acuta Presl in Sternberg, 1838 (a synonym of Bergeria dilatata - The type of the genus remains the holotype of Bergeria acuta). p 1836 Pachyphloeus Göppert, p. 433, 468 (Göppert's Pachyphloeus also includes one specimen with a large ulodendroid branch scar and several decorticated, indeterminable stem fragments).

1838 Bergeria Presl in Sternberg, p. 138.

Diagnosis. After Álvarez-Vázquez \& Wagner (2014). Arborescent lycopsid stems covered with spirally arranged, rhomboidal to fusiform leaf cushions, longer than broad, 
contiguous or separated by narrow grooves, without a differentiated leaf scar. Leaves long, grass-like to linearlanceolate, entire, single-veined.

Remarks. As previously mentioned by ÁlvarezVázquez \& Wagner (2014), Bergeria encompasses lycopsid specimens that lack a differentiated leaf scar. When leaves are preserved, they occupy the upper part of leaf cushions, to which they are attached by their entire width. If leaves are detached, a small, irregular scar marks the position of the leaf base at the top of leaf cushion; these would be the "false leaf scars" of Chaloner \& Boureau (in Boureau, 1967, p. 533).

However, since the beginning of the last century, the name Bergeria has commonly been applied to decorticated stems of lycopsids showing lepidodendroid leaf cushions. This has been in error. While Brongniart (1838, p. 35) recognized that both Ulodendron (a related genus) and Bothrodendron only represented different states of preservation of Lepidodendron, the error with respect to Bergeria probably originated with Fischer (1905), who figured and described as Bergeria adpression material with subepidermal preservation, thus lacking the outline of leaf scars. Fischer (1905, figs 5, 7) seems to have confused partially decorticated remains with better-preserved specimens possessing a false leaf scar (Fischer, 1905, fig. 6). This seems to be the reason why Fischer included type specimens of Bergeria acuta, the type species of the genus, with decorticated remains. The error was perpetuated by later authors.

Álvarez-Vázquez \& Wagner (2014) interpreted the type specimens of Bergeria acuta (Presl in Sternberg, 1838, Taf. XLVIII, figs 1a, 1b; Kvaček \& Straková, 1997, pl. 2 , figs 3,4 ) as being well-preserved stem fragments that are not decorticated and show the outline of leaf cushions as well as an irregular, poorly defined scar at the top of the leaf cushion. Previously, Lindley \& Hutton (1831, pl. 7, fig. 2; copy in Álvarez-Vázquez \& Wagner, 2014, fig. 13; here Fig. 2) had introduced Lepidodendron dilatatum, illustrated by a drawing of a single specimen displaying the same cushion characters as Presl's Bergeria acuta, and showing leaves in attachment. Lepidodendron dilatatum was introduced earlier than Bergeria acuta, so that the correct name for the species is Bergeria dilatata, as pointed out by Álvarez-Vázquez \& Wagner (2014).

Ulodendron was introduced by Lindley \& Hutton (1831) to include stem adpressions bearing large, subcircular branch scars showing an umbilicus in its central part; these scars are placed in vertical rows. Two species were initially distinguished, viz. Ulodendron majus (Lindley \& Hutton, 1831, pl. 5), based on a specimen from the Bensham coal seam, Jarrow Colliery, Newcastleupon-Tyne (Duckmantian), possessing broader than long leaf cushions and large, well-separated branch scars; and Ulodendron minus (Lindley \& Hutton, 1831, pl. 6), with rhomboidal, more equidimensional leaf cushions and smaller, closely spaced (separated by less than $1 \mathrm{~cm}$ ) branch scars, originating from the High Main coal seam, South Shields Colliery, Durham (also Duckmantian). Andrews (1955) designated Ulodendron majus as the genotype.

References to Ulodendron most commonly focus on the nature of the branch scars (corresponding either to strobilar or branch detachment), and their taxonomic significance. However, Ulodendron has been used in very different ways, and most species are represented only by their type specimens. Kidston (1885) reviewed the various references that existed until that date and concluded that Ulodendron majus and Ulodendron minus would be synonymous with Sigillaria discophora (Koenig) Kidston. He also included Lindley \& Hutton's (1833) Bothrodendron punctatum in the synonymy. Bureau (1913-14), when figuring several specimens with ulodendroid scars, rightly regarded the absence of leaf scars (i.e. permanent leaves) as the most important difference between Ulodendron and Lepidodendron.

On the other hand, Jonker (1976) synonymized both Ulodendron majus and Ulodendron minus with Lepidophloios laricinus. Ulodendroid scars are, in fact, rather common in Lepidophloios. We concur with Jonker's attribution of Ulodendron majus and Ulodendron minus to Lepidophloios, as defined on adpression material, but believe that two different species are involved. Since a number of arborescent lycopsids of Pennsylvanian age possessed branch scars, occurring either singly or in vertical rows, the presence or absence of such scars cannot be used for a generic distinction.

Thomas (1967) emended the diagnosis of Ulodendron so as to include cuticular characteristics. His study was based on the epidermal character of specimens, which he determined as Ulodendron majus; he did not investigate this character in Lindley \& Hutton's type material. According to Thomas (1967), the genus Ulodendron would be characterised by rhomboidal leaf cushions, broader than long, the presence of persistent linear leaves and large circular scars situated in vertical rows. This redefinition of the genus, based on cuticular material from a fragmentary and incompletely illustrated specimen, appears to generate more confusion around the genus, requiring systematic analysis beyond the scope of the present work. The diagnostic characteristics used by Thomas (1967) are such as to rule out any confusion with Bergeria; of particular relevance are the relative dimensions of the leaf cushions and the presence of a ligule pit used as diagnostic for Ulodendron. However, confusion has arisen from the redesignation by Thomas (1968) of material labelled as Lepidodendron landsburgii Kidston to Ulodendron. This latter species is here considered synonymous with Bergeria dilatata; not surprisingly Thomas's (1968) description of this material is closely similar to that recognised here 
for the species. The assignment of Kidston's species to Ulodendron is here considered incorrect.

DiMichele (1980) noted the similarities between the permineralized genus Paralycopodites, characterized by persistent leaves, and the adpression genus Ulodendron (sensu Thomas), but kept these two genera as separate taxonomic entities in view of the absence of anatomical characters in Ulodendron. According to DiMichele (1980), stems of Paralycopodites are frequently associated with bisporangiate strobili showing microsporangia in the upper part and megasporangia in the lower part (i.e., a Flemingites type cone).

In contrast, Pearson (1986) synonymized both Ulodendron and Paralycopodites with Anabathra (Witham, 1833), a genus based on a single specimen showing both external characters and internal anatomy. The holotype of the type species, Anabathra pulcherrima Witham, from uppermost Tournaisian/Viséan strata of Allanbank, Berwickshire, Scotland, was fully documented by Pearson (1986), who concluded that it is conspecific with Paralycopodites brevifolius (Williamson) DiMichele. Since the latter is a synonym of the type species, Paralycopodites minutissimum Morey \& Morey, Pearson placed Paralycopodites in synonymy with Anabathra, including it in the Flemingitaceae of Thomas \& BrackHanes (1984). Subsequently, DiMichele \& Phillips (1994, p. 59) restricted Anabathra to the type material; they regarded the type specimen of Anabathra as being too fragmentary to justify its synonymy with Paralycopodites. This may be regarded as a viable argument, but has no direct relevance to the nomenclature of material preserved as adpressions (as the one studied here).

Comparisons. Lepidodendron Sternberg is distinguished by its vertically elongate, rhomboidal or fusiform leaf cushions. Leaf scars are situated either centrally or on the upper half of the cushions, and show a leaf trace as well as two foliar parichnos; two infrafoliar parichnos are also present, as is a ligule pit above the scar. It is rare to find specimens with attached leaves.

The Namurian genus Meyenodendron (Thomas \& Spicer, 1986) possesses persistent leaves attached to the upper part of leaf cushions. However, cushions are obovate, with rounded upper and lateral angles and an infrafoliar bladder in the upper half of the cushion. There is no parichnos, and a ligule is visible in its upper part.

The Mississipian genus Tomiodendron (Radczenko, 1956; Meyen, 1972, 1976), initially described from Angaraland, includes ligulate stems with longitudinally elongate leaf cushions showing a small, subtriangular leaf scar in its upper part and a vascular trace in the centre. Attached, spirally arranged, short, falcate leaves have been reported for the Viséan Tomiodendron variabilis by Thomas \& Purdy (1982).
The Permian, Gondwanaland genus Brasilodendron (Chaloner et al., 1979; Spiekermann et al., 2018) is characterized by persistent leaves attached to fusiform leaf cushions. Brasilodendron leaves are attached to the middle part of the cushions, not at the cushion apex as in Bergeria. Also, leaves have a sigmoidal profile: they are first attached perpendicularly to the stem, then turning parallel to the axis, and the distal part is finally directed away from the stem. In addition, leaf margins show irregular fimbriae.

\section{Bergeria dilatata (Lindley \& Hutton, 1831) Álvarez- Vázquez \& Wagner, 2014}

(Figs 2-10b)

* v 1831 Lepidodendron dilatatum Lindley \& Hutton, p. 27, pl. 7, fig. 2 (here Fig. 2).

* 1831 Lepidodendron gracile Lindley \& Hutton, p. 30, pl. 9, figs 1, 2 (included in Lepidodendron ophiurus by Kidston, 1891, and in Bergeria dilatata by ÁlvarezVázquez \& Wagner, 2014).

1831 Lepidodendron Sternbergii, Lindley \& Hutton, p. 15-21, pl. 4.

p 1834 Lepidodendron Sternbergii, Lindley \& Hutton, p. 83, pl. 112, figs B, C; non pl. 112, fig. A (= Lepidophloios laricinus).

v p 1834 Lepidodendron selaginoides, Lindley \& Hutton, p. 85-86, pl. 113 (the specimen figured as Lepidodendron selaginoides by Lindley \& Hutton, 1831 , pl. 12 has not been included in our list of synonymy since its revision in the collections of the Hancock Museum has allowed verification that it must be included in Bothrodendron minutifolium).

v 1834 Lepidodendron elegans, Lindley \& Hutton, p. 99-100, pl. 118 (included in the synonymy of Lepidodendron similis by Kidston in Jongmans, 1909) (see Figs 3, 4).

p 1834 Lepidodendron Sternbergii, Mammatt, pl. 63, fig. 144a; non pl. A9 (cushions with leaf scars).

? 1834 Lepidodendron Dilatatum, Mammatt, pl. 75, fig. 8; pl. 77, fig. 10; pl. A2 (cannot be judged correctly from the diagrammatic drawings).

? 1834 Lepidodendron Dichotomum, Mammatt, pl. A3.

p 1836 Pachyphloeus tetragonus Göppert, p. 433, 468, Tab. XLIII (cited as Tab. XLI in the text), fig. 2 (figured upside down); non Tab. XLIII, figs 1, 3, 4 (decorticated, indeterminable stems?); non Tab. XLIII, fig. 5 (ulodendroid branch scar).

* 1838 Bergeria acuta Presl in Sternberg, p. 184, Taf. XLVIII, figs 1a, 1b (acc. to Álvarez-Vázquez \& Wagner, 2014). 
* 1838 Bergeria angulata Presl in Sternberg, p. 184, Taf. LXVIII, fig. 17 (synonym of Lepidodendron acutum, acc. to Němejc, 1947; to Bergeria dilatata acc. to Álvarez-Vázquez \& Wagner, 2014).

* 1838 Bergeria marginata Presl in Sternberg, p. 184, Taf. LXVIII, fig. 16 (synonym of Lepidodendron acutum, acc. to Němejc, 1947; to Bergeria dilatata acc. to Álvarez-Vázquez \& Wagner, 2014).

* 1838 Bergeria quadrata Presl in Sternberg, p. 184, Taf. LXVIII, fig. 19 (Lepidodendron acutum, acc. to Němejc, 1947; to Bergeria dilatata acc. to ÁlvarezVázquez \& Wagner, 2014).

* 1838 Bergeria rhombica Presl in Sternberg, p. 184, Taf. LXVIII, fig. 18 (synonym of Lepidodendron acutum, acc. to Němejc, 1947; to Bergeria dilatata acc. to Álvarez-Vázquez \& Wagner, 2014).

? 1838 Lepidodendron elegans, Brongniart, p. 85, pl. 14, figs 1-2A (two specimens with medium-sized, repeatedly dichotomized, leafy branches. The enlarged image shows leaf scars, so a revision of the original material would be needed).

1838 Lepidodendron, Brongniart, pl. 30, figs 1-2A (two specimens with attached leaves).

1848 Lepidodendron dilatatum, Sauveur, pl. LX, fig. 3 (stem fragment with obovate cushions and long leaves similar to holotype).

* 1848 Lepidodendron gibbosum Sauveur, pl. LX, fig. 4.

1848 Lepidodendron sternbergii, Sauveur, pl. LIX, fig. 1 (dichotomized branches).

1852 Lepidodendron dilatatum, Owen, Tab. VI, fig. 5. 1852 Lepidodendron obovatum, Owen, Tab. VI, fig. 2. * 1854 Lepidodendron Haidingeri von Ettingshausen, p. 55, Taf. 22; Taf. 23, figs 1,2 (acc. to ÁlvarezVázquez \& Wagner, 2014).

? 1855 Lepidodendron elegans, Phillips, p. 235, fig. 115.

* 1859-60 Knorria apicalis Eichwald, p. 154-155, pl. XII, fig. 1.

* p 1859-60 Sagenaria Glincana Eichwald, p. 127130, pl. V, fig. 21 (dichotomized branches); pl. Va, figs 6, 7; non pl. V, fig. 22 (decorticated); non pl. Va, figs $1-3,5$ (= "Lepidodendron" rimosum); non pl. Va, fig. 4 (decorticated).

p 1859-60 Sagenaria undulata, Eichwald, p. 126-127, pl. VIII, fig. 8; non pl. IX, fig. 1 (difficult to judge).

* 1859-60 Ulodendron pumilum Eichwald, p. 144-146, pl. X, fig. 5 (with ulodendroid branch scar).
* p 1859-60 Ulodendron transversum Eichwald, p. 139140, pl. IX, fig. 8 (with ulodendroid branch scar); non pl. VI, fig. 13 (decorticated).

* 1860 Lepidodendron Oweni Wood, p. 239, pl. 5, fig. 1 (acc. to Álvarez-Vázquez \& Wagner, 2014).

1868 Lepidodendron dilatatum, von Roehl, p. 134, Taf. VIII, fig. 4.

1870-74 Lepidodendron Sternbergii, Schimper, p. 19, pl. LVIII, figs 1-4.

1875 Sagenaria elegans, Feistmantel, p. 203-204, Taf. XXXVII, fig. 3.

1875 Bergeria rhombica, Feistmantel, p. 200-201, Taf. XLI, figs 3-4a.

1878 Lepidodendron lycopodioides, Zeiller, pl. CLXXI (large stem fragment and small leafy branches, isotomously forked, similar to that figured as Lepidodendron elegans by Lindley \& Hutton, 1834, pl. 118).

* 1879-80 Lepidodendron lanceolatum Lesquereux, $\mathrm{p}$. 369, pl. LXIII, figs 3-5.

* ? 1879-80 Lepidodendron Scutatum Lesquereux, p. 369 , pl. LXIII, figs 6-6c (presence or absence of leaf scars is not clear from the drawings - acc. to ÁlvarezVázquez \& Wagner, 2014).

1879-80 Lepidodendron rhombicum, Lesquereux, p. 382, pl. LXII, figs 4, 4a; pl. LXIV, fig. 18.

? 1884 Lepidodendron (Bergeria) marginatum, Lesquereux, p. 784, pl. CVII, fig. 3.

p 1886-88 Lepidodendron Haidingeri, Zeiller, p. 461463, pl. LXIX, fig. 1; non pl. LXIX, fig. 1A (drawing which shows a leaf scar).

1886-88 Lepidodendron lycopodioïdes, Zeiller, p. 464 467, pl. LXIX, figs 2-3A; pl. LXX, fig. 1 (same as Zeiller, 1878, pl. CLXXI; included by Kidston, 1911 in his Lepidodendron simile).

p 1886-88 Lepidodendron ophiurus, Zeiller, p. 458461, pl. LXVIII, figs 1-1A, figs 4-6A; non pl. LXVIII, figs 2, 3 (strobili).

? 1886-88 Ulodendron majus, Zeiller, p. 481-483, pl. LXXIII, fig. 1 (although leaf cushions shape and other features seem to correspond those of Bergeria dilatata, Zeiller mentioned leaves $250 \mathrm{~mm}$ long, much longer than those in our specimens).

? 1887 Lepidodendron lanceolatum, Kidston, p. 394, pl. XXVII, fig. 5 (small branch with strobilus); pl. XXVIII, figs 3, 4. 


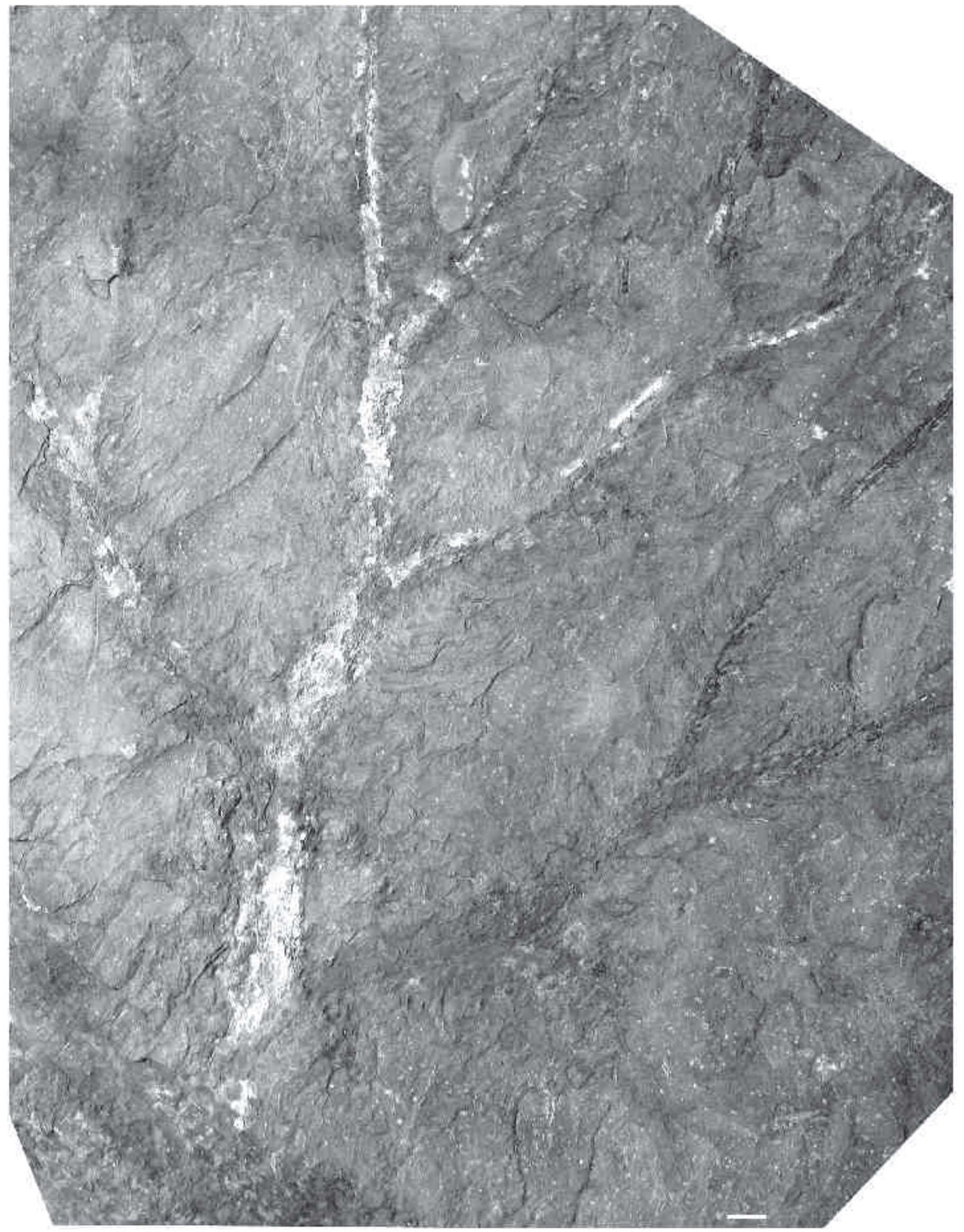


p 1888 Lepidodendron Sternbergii, Howse, p. 78, 79, 80, 81 (Lepidodendron dilatatum quoted as a synonym).

* 1893 Lepidodendron Landsburgii Kidston, p. 338339, pl. III, figs 9-9a; pl. III, figs 10-10b (transferred to Ulodendron by Thomas, 1968; to Anabathra by Pearson, 1986; and regarded as synonymous with Bergeria dilatata by Álvarez-Vázquez \& Wagner, 2014).

1899 Lepidodendron lanceolatum, White, p. 192-195, pl. LIII, figs 2-2a.

1903a Lepidodendron lycopodioides, Arber, p. 12, pl. II, fig. 5.

1903b Lepidodendron lycopodioides, Arber, p. 19, textfig. (without number).

p 1903 Lepidodendron Glincanum, Kidston, pl. III, figs 27, 28; non p. 762-765, pl. II, figs 20, 21 (= Lepidodendron canobianum Crookall acc. to Crookall, 1964); pl. IV, figs 37-40 (= Lepidodendron canobianum); pl. V, figs 41-31 (= Lepidodendron canobianum).

1904 Lepidodendron ophiurus, Zalessky, p. 23-25 [9596], pl. V, figs 1-3, fig. 4 (leafy twigs), figs 6-7.

1904 Ulodendron sp., Zalessky, p. 36-37 [101-102], pl. VI, fig. 5 (medium-sized branch with attached leaves); text-figs 7-8.

* ? 1907 Lepidodendron Tonderae Zalessky, p. 28-29 [60-61], Taf. I, fig. 7 (cushions with a well-marked keel); text-fig. 6 (drawing).

1907 Ulodendron sp., Zalessky, p. 29-30 [61], textfigs 7,8 .

p 1909 Lepidodendron similis Kidston in Jongmans, p. 174, 201, 215 (nomen nudum - Although Lepidodendron simile is very often cited and figured as a separate species, the specific name similis was published without a proper diagnosis, description or figuration. Only a list of synonymy was provided: 1) Lepidodendron elegans, Lindley \& Hutton, 1834, pl. 118 - here included in Bergeria dilatata; 2) Lepidodendron elegans, Brongniart, 1838, pl. 14, figs 1-2A - also included here, with doubt, in Bergeria dilatata); 3) Lepidodendron lycopodioides, Zeiller, 1878, pl. CLXXI - here included in Bergeria dilatata; 4) Lepidodendron lycopodioides,
Renault, 1882, pl. 5, fig. 8 - cushions with leaf scars and short, well-marked transverse lines in the lower part of leaf cushions; and 5) Lepidodendron lycopodioides, Zeiller, 1886, pl. LXIX, figs 2-3A; pl. LXX, fig. 1 here included in Bergeria dilatata).

p 1911 Lepidodendron simile Kidston, p. 137-138 (excluding Renault's 1882, pl. 5, fig. 8 - Kidston repeated his 1909 list of synonymy, except Lindley \& Hutton's illustration of Lepidodendron elegans which he compared at that time with Lepidodendron lycopodioides. A proper diagnosis, description or illustration is lacking).

? 1912 Lepidodendron lanceolatum, Arber, p. 250, pl. 12 , fig. 14 (with short, well-marked transverse lines in lower part of leaf cushions).

* 1913-14 Lycopodites foliosus Bureau, p. 160, pl. XXIX, figs 1-3.

p ? 1913-14 Lepidodendron lycopodioides, Bureau, p. 118-121; pl. XXXI, fig. 1; pl. XXXII, fig. 1; pl. XXXIII, figs 1-2 (small branches bearing strobili); pl. XXXIV, figs 1-2 (leafy twigs); non pl. XXVIII, fig. 5 (?); non pl. XXXIII, fig. 3 (strobilus); non pl. XXXIV, figs 3-6 (strobili).

p 1913-14 Lepidodendron Veltheimianum, Bureau, p. 136-142, pl. XXX ${ }^{\mathrm{BIS}}$, figs 1-1B (leafy twigs isotomously forked at acute angles - Bureau also mentioned Lepidodendron lycopodioides in association); pl. XXXI, figs 2-3 (leafy twigs); non pl. II, fig. 6 (decorticated specimen resembling Lepidodendron lossenii Weiss); non pl. IV, fig. 1 (to be compared with Lepidodendron lossenii); non pl. XXXIX, figs 4-4A (= Lepidodendron veltheimii Sternberg); non p1. XL, figs 4-4A (= Lepidodendron veltheimii); non pl. XLIII, figs 1-1B (= Lepidodendron lossenii), fig. 2 (indeterminable, decorticated specimen); non pl. XLIV, figs 1-2 (decorticated), fig. 3 (too fragmentary to be identified specifically); ? non pl. XLV, figs 1-2 (ulodendroid branch scars).

? 1914 Lepidodendron lanceolatum, Arber, p. 402, pl. 28, fig. 26 (short transverse markings in the lower part of leaf cushions).

1916 Bergeria angulata, Hörich, p. 428, fig. 3 (photograph of Presl's 1838, Taf. LXVIII, fig. 17).

Figure 3. Bergeria dilatata (Lindey \& Hutton) Álvarez-Vázquez \& Wagner. HM G02.17 (x 0.75). Large stem (lower left corner) with leafy twigs branching isotomously at acute angle. Part of the specimen figured as Lepidodendron elegans by Lindley \& Hutton (1834, pl. 118 - Fig. 4 here). Note the resemblance with the stem fragment with attached leaves and small leafy branches figured as Lepidodendron lycopodioides by Zeiller (1878, pl. CLXXI; 1886, pl. LXX, fig. 1). Origin: Low Main coal seam, Felling Colliery, Newcastle-upon-Tyne; lower Duckmantian. Scale bar $=1 \mathrm{~cm}$. Repository: Great North MuseumHancock, Newcastle upon Tyne, England. 


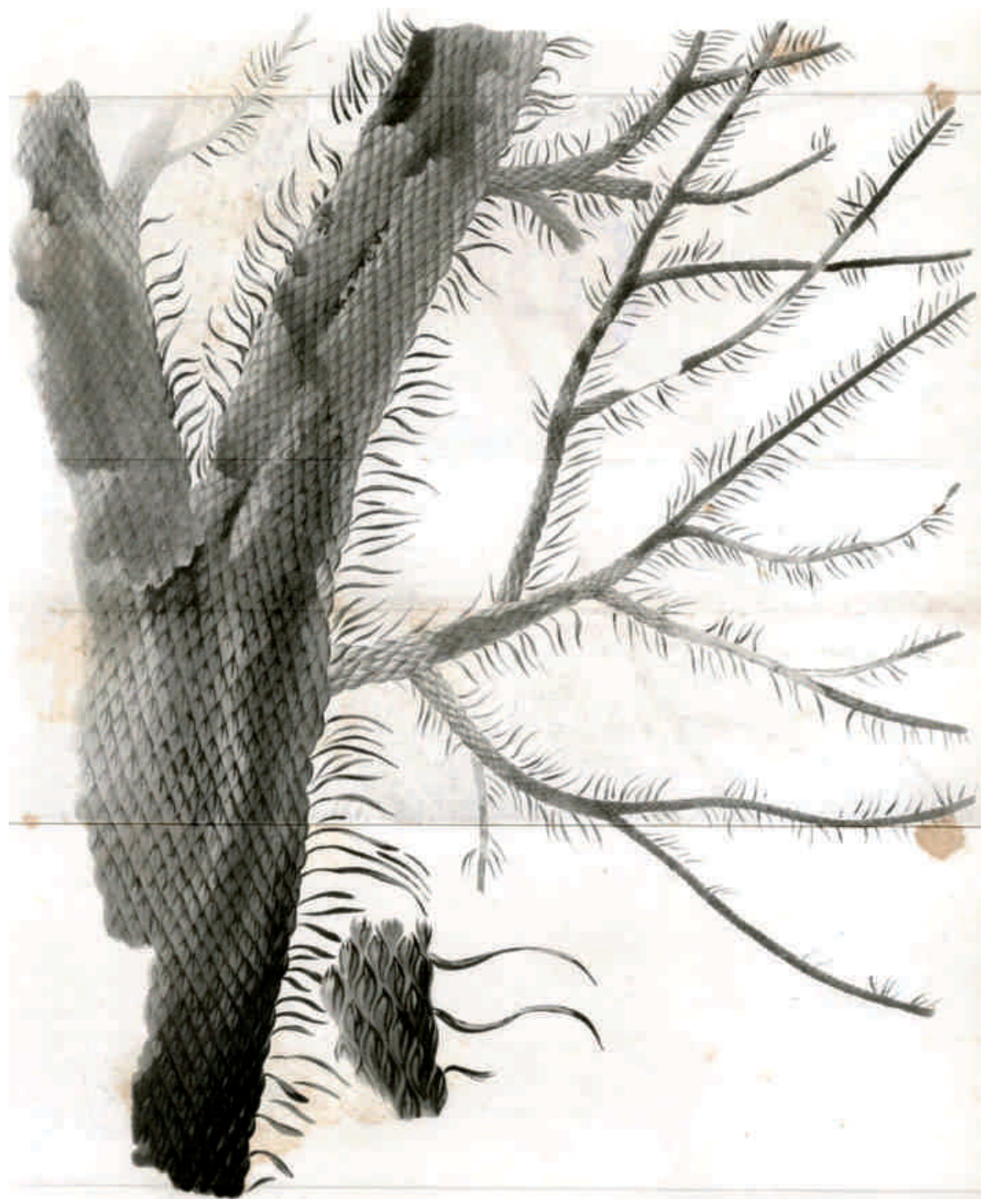

Figure 4. Copy, reduced to less than half, of the original figure published by Lindley \& Hutton (1834, pl. 118) as Lepidodendron elegans. 
1922 Lepidodendron lycopodioides, Arber, p. 191-195, pl. 10, figs 1-9; pl. 11, figs 10, 11, fig. 12 (cf.), fig. 13, fig. 14 (with doubt), figs 15A-16.

1922 Lepidodendron lanceolatum (= L. lycopodioides), Arber, p. 195-196, pl. 11, fig. 17; pl. 12, figs 18-22.

1925 Lepidodendron simile, Crookall, p. 391, pl. XVI, fig. 2.

1928 Lepidodendron sp., Šusta, Taf. VIII, Abb. 2 (small branches with associated strobili); Taf. LVIII, Abb. 4; Taf. LXII, Abb. 1.

1929 Lepidodendron lanceolatum, Crookall, pl. XX, fig. a.

? 1929 Lepidodendron acutum, Crookall, pl. XX, fig. f (small branches with strobili - difficult to judge from the illustration).

1929 Lepidodendron simile, Crookall, pl. XX, fig. d (same as Crookall, 1925, pl. XVI, fig. 2).

1929 Lepidodendron lycopodioides, Gothan \& Franke, p. 73, Taf. 30.2 (drawing).

1932 Lepidodendron lycopodioides, Crookall, pl. VI, fig. 5.

1932 Lepidodendron ophiurus, Crookall, pl. VII, fig. 4 (leafy twigs; difficult to judge from the illustration at natural size).

* 1934 Lepidodendron adygense Zalessky, p. $4-5$ (1617), 20, 21, pl. II, fig. 5; pl. IX, figs 2-2a.

1937 Lepidodendron ophiurus, Jongmans, p. 397, pl. 15 , figs 23-24.

1938 Ulodendron lycopodioides, Renier \& Stockmans in Renier et al., p. 63, pl. 15 (in association with one strobilus attributed to Ulostrobus geinitzii).

? 1938 Lepidodendron lycopodioides, Bell, p. 93-94, pl. XCVI, fig. 2, fig. 3 (presence or absence of leaf scars is unclear); pl. XCVII, figs 1-3 (small leafy branches).

p 1940 Lepidodendron ophiurus, Bell, p. 123, pl. VII, figs 5, 7; non pl. VII, fig. 6 (indeterminable; single leaf cushion with attached leaf).

1942 Lepidodendron simile, Němejc, p. 140, Tab. IV, fig. 2; Tab. IV, figs 3, 4 (with doubt - difficult to judge from the illustration).

v 1944 Lepidodendron lanceolatum, Bell, p. 88-89, pl. XLVIII, fig. 3 (leafy twigs with terminal strobili).

p 1947 Lepidodendron simile, Němejc, p. 68-72, Tab. I, figs 9-10; non Tab. I, fig. 1 (= "Lepidodendron" ophiurus), figs 2-3 (= "Lepidodendron" ophiurus?).

1949 Lepidodendron lanceolatum, Arnold, p. 165-167, pl. V; pl. VI, figs 4-6. p 1949 Lepidodendron ophiurioides?, Arnold, pl. IV, fig. 5; non p. 162-165, pl. III, fig. 4 (= Lepidodendron ophiurioides); pl. IV, figs 1-3 (= Lepidodendron ophiurioides) (Arnold compared with "Lepidodendron" ophiurus and Lepidodendron lycopodioides).

1949 Lepidodendron vestitum, Arnold, p. 168-169, pl. II, fig. 5.

1949 Lepidodendron ophiurus, Jongmans, p. 41, Afb. 1.25 (profusely branched specimen).

1951 Lepidodendron cf. ophiurus, Jongmans, p. 11, 12, pl. III, figs 22-22a, 29-29a.

1951 Lepidodendron groupe ophiurus, Jongmans, p. 21, 23, pl. XI, fig. 97; pl. XII, figs 98-99a (leafy branches); pl. XV, fig. 133.

? 1951 Lepidodendron ophiurus, Jongmans, p. 22, pl. XIV, fig. 123 (leafy twigs which cannot be judged adequately from the illustration).

1952 Lepidodendron acutum, Jongmans, p. 8, pl. II, figs 13-16 (leafy branches); pl. XIII, fig. 97 (leafy branches).

p 1952 Lepidodendron obovatum, Jongmans, p. 8, pl. II, figs 17, 18; non pl. XVII, figs 136-136a (= Lepidophloios?); non pl. XX, fig. 151 (= Lepidodendron bellii).

p 1952 Lepidodendron obovatum, Novik, Tab. XXV, fig. 1; non Tab. XXV, fig. 2 (difficult to judge), fig. 3 (resembles Lepidodendron bellii).

1952 Lepidodendron adygense, Novik, p. 187, 459, pl. XXIX, figs 1, 2 (same as Zalessky, 1934, pl. IX, figs 2-2a).

* 1952 Ulodendron Goodei Stockmans \& Willière, pl. E, fig. 1 (associated with Ulostrobus goodei), pl. E, fig. 3 (three times isotomously forked leafy twigs); pl. F, fig. 2 (leafy twigs associated with Ulostrobus goodei). (Although Stockmans \& Willière's specimens are leafy twigs, which are difficult to judge properly from the illustrations, all originate from a locality that also yielded two specimens with small cushions identified as Ulodendron sp. by Stockmans \& Willière and which we here assigned to Bergeria dilatata - see below).

1952 Ulodendron sp., Stockmans \& Willière, pl. E, figs 4,5 .

1953 Lepidodendron ophiurus, Jongmans, p. 39, pl. 2 , figs 9-10a; pl. 3, figs 11-11a (leaf cushions with short wrinkles in the lower part), figs 12-12a.

1953 Ulodendron sp., Stockmans \& Willière, pl. XXIV, fig. 7.

1954 Lepidodendron lycopodioides, Novik, p. 50-51, 134, Tab. X, fig. 1. 

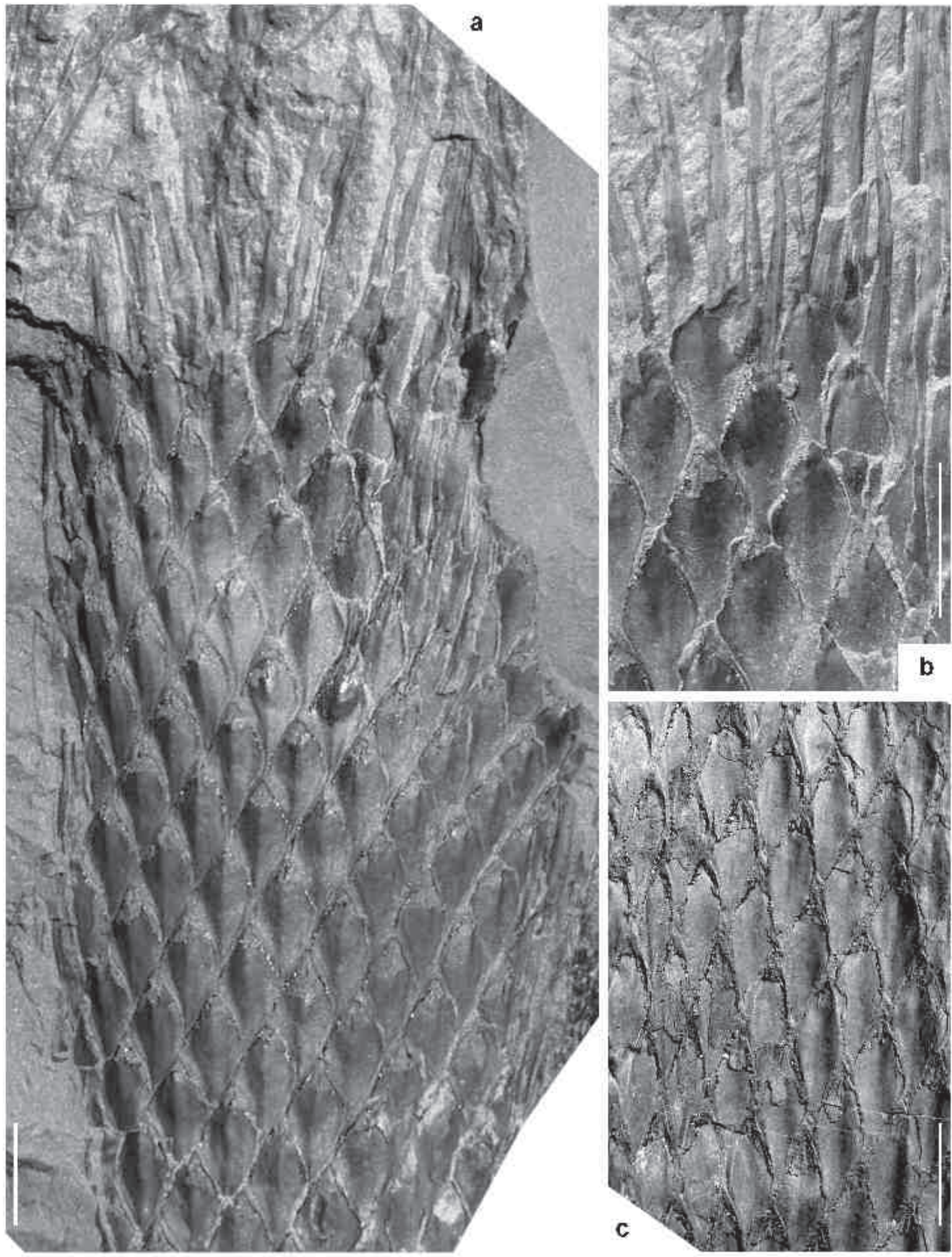
1957 Lepidodendron lycopodioides, Gothan \& Remy, p. 67, Abb. 57.

v 1959 Lepidodendron ophiurus, Jongmans in Wagner, p. 399.

1959 Sublepidodendron lycopodioides, Remy \& Remy, p. 100, 103, Abb. 79a-79b.

v 1960 Lepidodendron simile, Wagner, p. 82, 84-86, figs 22c, 23a, 24a-24f.

p 1962 Lepidodendron bretonense, Bell, pl. XLIX, fig. 2; non p. 53-54, pl. XLVII, fig. 5 (= Diaphorodendron decurtatum acc. to Álvarez-Vázquez \& Wagner, 2014), fig. 6 (decorticated specimen comparable with "Lepidodendron" rimosum acc. to ÁlvarezVázquez \& Wagner, 2014); non pl. XLVIII, fig. 4 (= Diaphorodendron decurtatum), fig. 6 (specimen with elongate, fusiform leaf cushions comparable with "Lepidodendron" rimosum).

p 1962 Lepidodendron pictoense, Bell, p. 52-53, pl. XLVI, fig. 1; pl. XLIX, fig. 3; pl. L, figs 1-3; non pl. XLIX, fig. 1.

1964 Lepidodendron acutum, Crookall, p. 285-287, pl. LX, fig. 2 (leaf cushions with barely distinct keels crossed with short wrinkles, showing also attached leaves); text-fig. 92 (copy of Presl in Sternberg, 1838, Taf. XLVIII, fig. 1b).

p 1964 Lepidodendron ophiurus, Crookall, pl. LXI, fig. 4 (several times dichotomized leafy twigs); pl. LXI, fig. 9 (same as Crookall, 1932, pl. VI, fig. 5); pl. LXIII, figs 1, 2 (syntypes of Lepidodendron landsburgii); non $\mathrm{p}$. 287-294, pl. LXII, fig. 5 (= "Lepidodendron" ophiurus); non pl. LXX, fig. 7 (compared with "Lepidodendron" volkmannianum by Álvarez-Vázquez \& Wagner, 2014); non pl. LXXIII, fig. 4 (branchlet with terminal strobilus); non text-fig. 93 (copy of "Lepidodendron" ophiurus Brongniart, 1822).

1964 Ulodendron sp., Drägert, p. 69, Taf. 12, fig. 1.

v 1965 Lepidodendron simile, Wagner, p. 17, pl. 9, fig. 11 (same as Wagner, 1960, fig. 24b).

1966 Lepidodendron ophiurus, Bell, pl. XX, fig. 1 (same as Bell, 1940, pl. VII, fig. 7).

1966 Lepidodendron pictoense (= ? L. ophiurus), Bell, pl. IV, fig. 13 (leafy twigs); pl. XXIX, fig. 1. p 1966 Lepidodendron lycopodioides, Greber, p. 108109, pl. XLVI, figs 1-1a; non pl. XLVI, figs 4-5 (cannot be judged from the illustration).

1967 Lepidodendron acutum, Chaloner \& Boureau in Boureau, p. 537.

p 1967 Lepidodendron simile, Chaloner \& Boureau in Boureau, p. 549, figs 377A-377B, fig. 379; non fig. 378 (leafy branches several times dichotomized associated with Flemingites dubius cones; previously figured in Chaloner, 1953).

k 1968 Ulodendron landsburgii, Thomas, p. 426-428, text-fig. A (drawing), text-figs C-D (drawings of cuticle).

? 1969 Lepidodendron, Daber, Taf. VIII, figs 1, 1a (difficult to judge).

1972a Lepidodendron obovatum, Biţoianu, pl. I, fig. 1 (figured upside down).

1972a Lepidodendron ophiurus, Biţoianu, pl. I, fig. 2.

1972a Lepidostrobus sp., Biţoianu, pl. III, fig. 26.

1972b Lepidodendron acutum, Bițoianu, pl. I, fig. 1 (same as Biţoianu, 1972a, pl. I, fig. 1).

? 1972b Lepidodendron obovatum, Biţoianu, pl. I, fig. 2 (difficult to judge from the poor illustration).

1972b Lepidostrobus sp., Biţoianu, pl. I, fig. 3 (same as Biţoianu, 1972a, pl. III, fig. 26).

1972 Lepidodendron subdichotomum, Maithy, p. 85, pl. 49 , fig. 1; text-fig. 2 A.

? 1972 Lepidodendron ophiurus, Maithy, p. 86, pl. 49, fig. 2; text-fig. 2B (specimen with short transverse marks in lower part of cushions).

1972 Lepidodendron lycopodioides, Migier, Tab. I, fig. 2.

1973 Lepidodendron acutum, Bassett \& Edwards, p. 14 (figure).

? 1973 Lepidodendron cf. lanceolatum, Bassett \& Edwards, p. 21 (figure below).

? 1973 Lepidodendron acutum, Biţoianu, pl. I, fig. 1 (same as Biţoianu, 1972b, pl. I, fig. 2).

1976 Ulodendroid scars of Lepidodendron aculeatum, Jonker, p. 111, pl. 1, figs 1, 2.

Figure 5. Bergeria dilatata (Lindey \& Hutton) Álvarez-Vázquez \& Wagner. a) PBE11000 (x 2). Large stem showing obovate, smooth cushions and long, entire, grass-like leaves inserted at the top. Figured (partly) as Ulodendron acutum by Wagner \& ÁlvarezVázquez (2010, pl. XI, fig. 2). Origin: Corta Ballesta; 2 m above Capa (Seam) 7 (locality 10251); lower Bolsovian. b) Another enlargement (x 3) of the upper part of the same specimen. Note the insertion of leaves in the uppermost part of leaf cushions. c) PBE09515 (x 2). Origin: borehole B4 in Aurora area, at 265,40 m depth (locality GIV-B4-M46); lower Bolsovian. Scale bars $=1 \mathrm{~cm}$. Repository: Centro Paleobotánico, Real Jardín Botánico de Córdoba, Spain. 


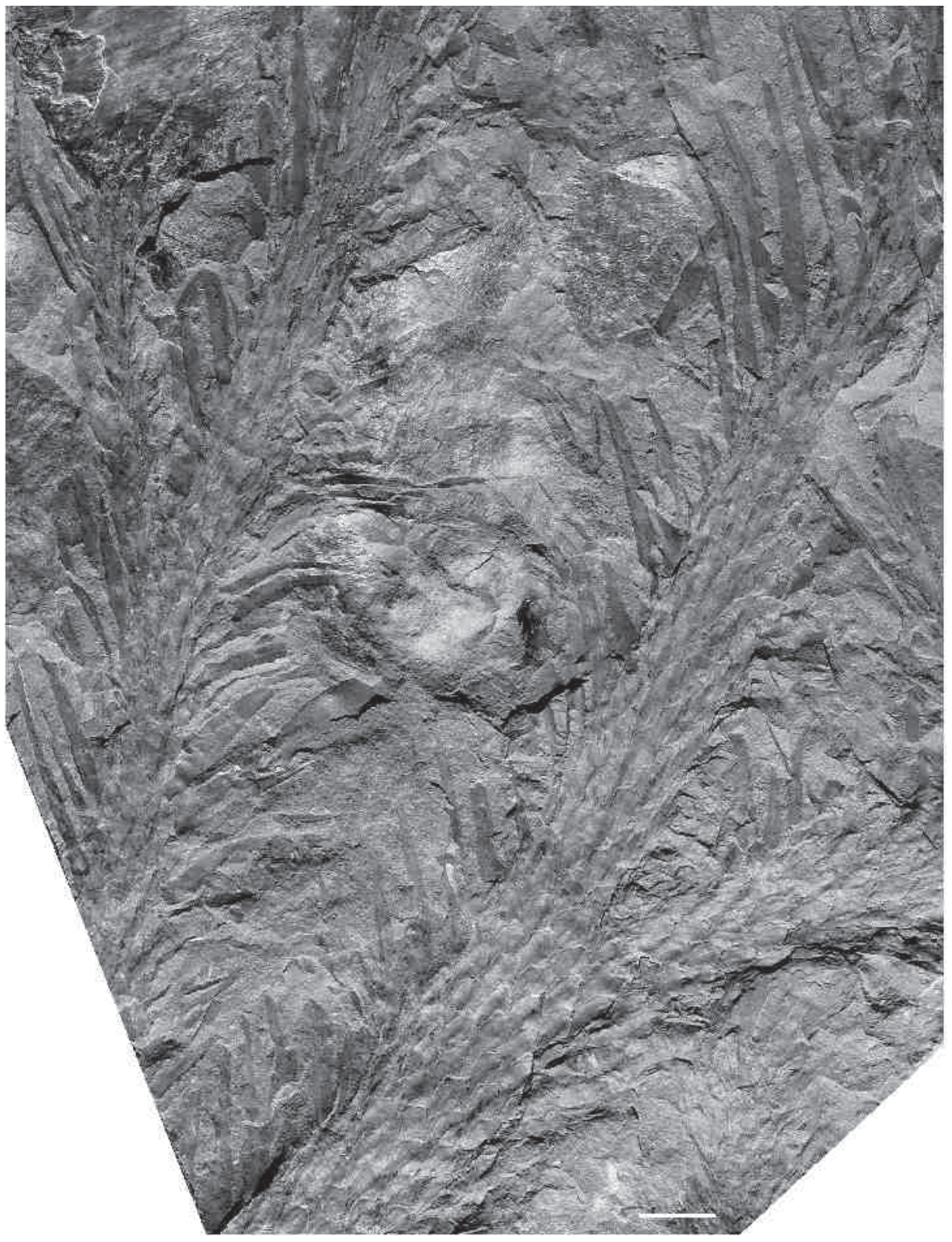


1977 Lepidodendron simile, Remy \& Remy, p. 315, Bild 184 (same as Gothan \& Remy, 1957, Abb. 57).

1978 Lepidodendron $\mathrm{cf}$. wortheni, Gillespie et al., p. 52, pl. 12, fig 2.

? 1978 Lepidodendron with attached Lepidophylloides, Gillespie et al., p. 45, 52, pl. 12, fig. 4; pl. 13, figs 3-5 (small branches with attached leaves; poorly figured).

1979 Lepidodendron lycopodioides, van Amerom \& Lambrecht, p. 153, Taf. VIII, figs 1a-1b (?), fig. 7.

1979 Lepidodendron adygense, Anisimova, p. 32-33, Tab. I, fig. 2.

? 1979 Lepidodendron lycopodioides, Anisimova, Tab. I, fig. 4 (poorly figured).

1979 Lepidodendron worthenii, Anisimova, Tab. II, fig. 3.

1981 Lepidodendron ophiurus, van Amerom, fig. 2.D.

? 1979 Lepidodendron simile, Purkyňová, p. 188, Tab. II, fig. 4.

1982 Lepidodendron acutum, Bassett \& Edwards, p. 18 (figure without number - associated with terminal strobili) (same as Bassett \& Edwards, 1973, p. 14).

? 1982 Lepidodendron cf. lanceolatum, Bassett \& Edwards, p. 26 (right) (same as partially reproduced by Bassett \& Edwards, 1973, p. 21).

? p 1982 Lepidodendron ophiurus, Migier, p. 38, 67, Tab. I, fig. 1, fig. 2; non Tab I, fig. 3 (=Asterophyllites?); non Tab. I, fig. 6 (difficult to judge).

1983 Lepidodendron acutum, Chaloner \& MeyerBerthaud, p. 138, 142, fig. 2.

? 1983 Lepidodendron cf. acutum, Chaloner \& MeyerBerthaud, fig. 4 (difficult to judge from the illustration).

1983 Lepidodendron simile, Chaloner \& MeyerBerthaud, p. 138, 141, 142, fig. 3 (same as Chaloner \& Boureau in Boureau, 1967, fig. 379).

1983 Lepidodendron simile, Rex \& Chaloner, p. 237, text-fig. 3D; text-fig. 3E (drawing of the previous specimen).

? 1983 Lepidodendron cf. aculeatum, Josten, Taf. 22, figs 1,2 (difficult to judge from illustrations at natural size).
? 1983 Lepidodendron aculeatum, Josten, p. 71 (excluding synonymy), Taf. 22, fig. 3 .

v 1983 Lepidodendron simile, Wagner, p. 158.

v 1983 Lepidodendron simile, Wagner in Wagner \& Bowman, p. 154.

v 1983 Lepidodendron simile, Wagner in Wagner et al., p. 61.

v 1984 Lepidodendron simile, Wagner in Wagner et al., p. 35.

? 1985 Lepidodendron acutum, Gillespie \& Crawford, p. 250, pl. I, fig. 3 (poorly figured).

? 1985 Lepidodendron cf. rimosum, Gillespie \& Crawford, p. 250, pl. I, fig. 6 (presence or absence of leaf scars is unclear).

? 1985 Lepidodendron acutum, Gillespie \& Rheams, p. 200, pl. III, fig. 9 (presence or absence of leaf scars is unclear).

1986 Lepidodendron acutum, Purkyňová, p. 59, Tab. III, figs 1-2.

1986 Lepidodendron, Thomas, p. 26, top figure.

1987 Lepidodendron acutum, Tenchov, p. 58, pl. XXI, figs 1-2.

p 1987 Lepidodendron lycopodioides, Tenchov, p. 60, pl. XXIV, figs 7, 9; non pl. XXIV, figs 6, 8 (= Diaphorodendron decurtatum).

p 1987 Lepidodendron obovatum, Tenchov, pl. XXIII, fig. 3; non p. 60, pl. XXIII, figs 1, 5, 6 (= Lepidodendron bellii); non pl. XXIII, fig. 2 (= Lepidodendron aculeatum), fig. 4 (= Lepidodendron aculeatum).

1988 Lepidodendron lycopodioides, Cleal \& Thomas, p. 418 , pl. 3, fig. 1 .

1989 Lepidodendron simile, Laveine, p. 30, pl. 4, figs 2-2a; text-fig. 20 (drawings).

1992 Lepidodendron acutum, Kvaček \& Kvaček, Tab. I, fig. 3 (partial illustration of Bergeria acuta Presl, 1838, Taf. XLVIII, fig. 1a).

1994 Lepidodendron acutum, Cleal \& Thomas, p. 103, tex-fig. 48 (drawing); text-fig. 49 (dichotomized medium-sized branches with attached leaves).

Figure 6. Bergeria dilatata (Lindey \& Hutton) Álvarez-Vázquez \& Wagner. PBE11001 (x 1.5). Medium-sized branches forked at acute angles. Note the strong resemblance with Lepidodendron haidingeri as figured by Ettingshausen (1854, Taf. 22) and Zeiller (1886, pl. LXIX, fig. 1), with Bureau's Lycopodites foliosus (1913, pl. LXXIX, figs 1-3), with the magnificent specimen figured as Lepidodendron acutum by Bassett \& Edwards (1973, 1982), and with the specimen figured as Lepidodendron by Thomas (1986). Origin: Corta Ballesta; 1,80 m above Capa (Seam) 13 (locality 10257); lower Bolsovian. Scale bar = $1 \mathrm{~cm}$. Repository: Centro Paleobotánico, Real Jardín Botánico de Córdoba, Spain. 

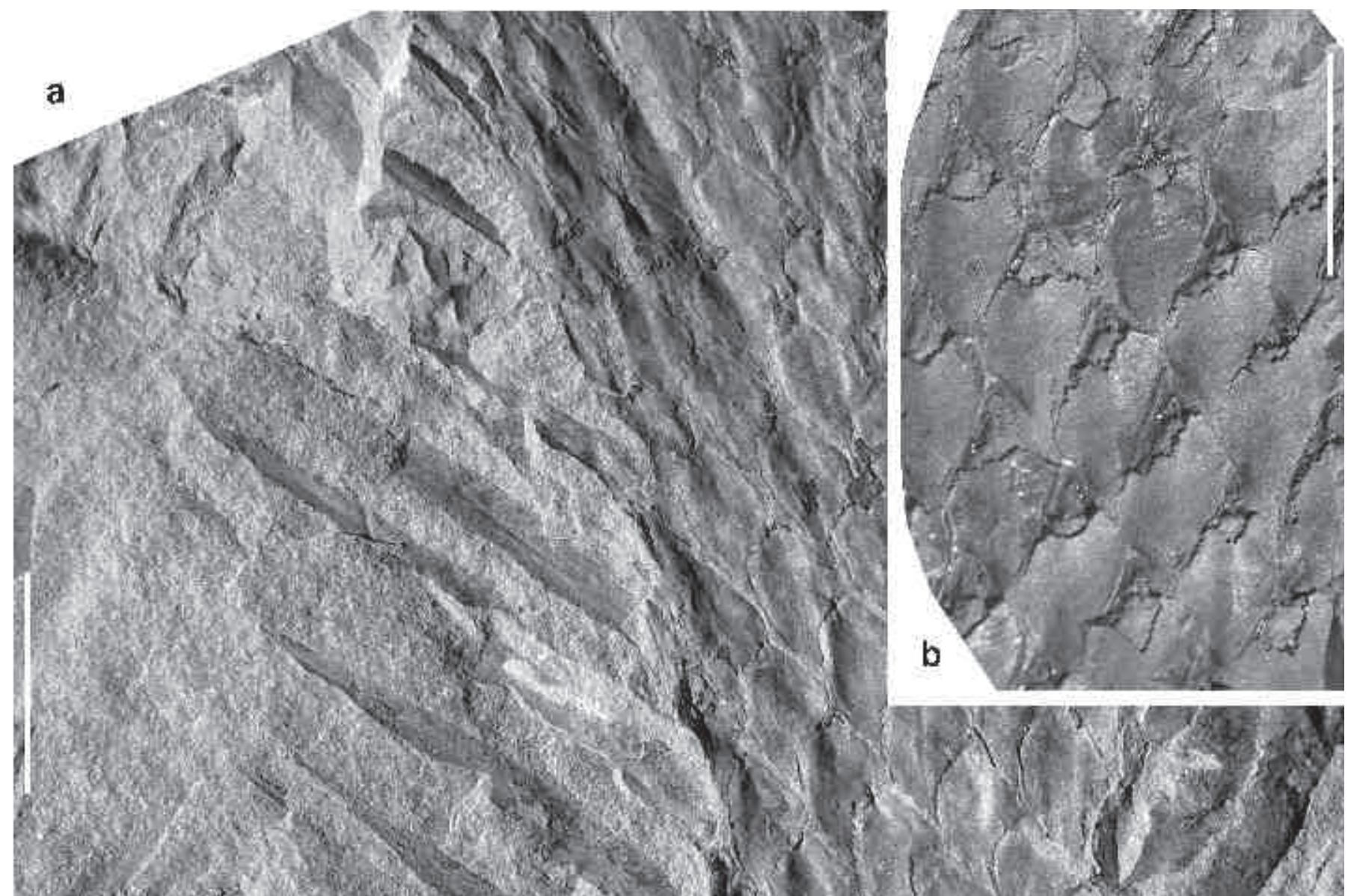

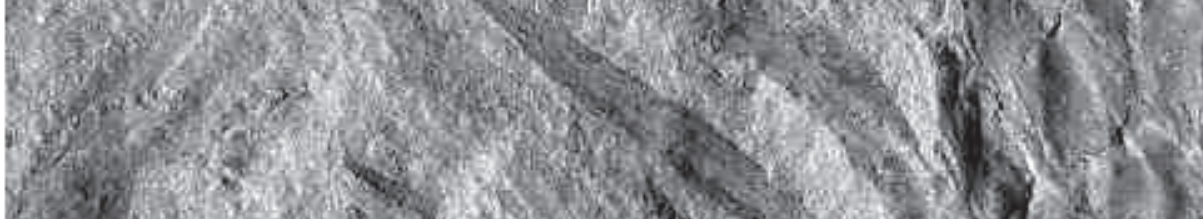

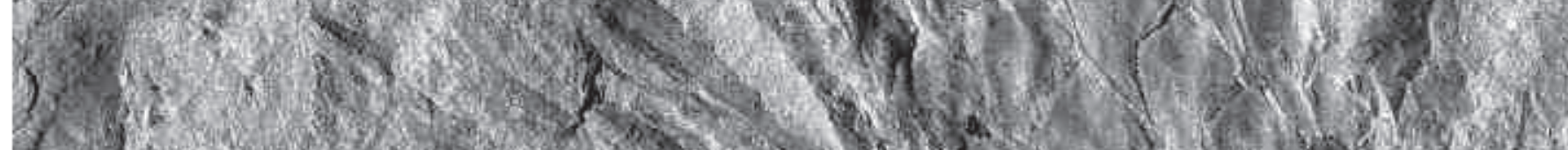

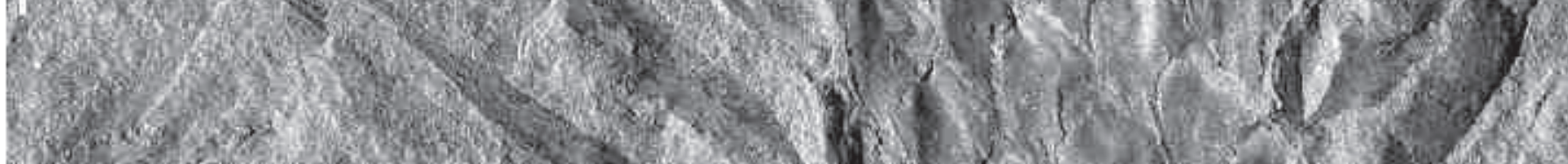
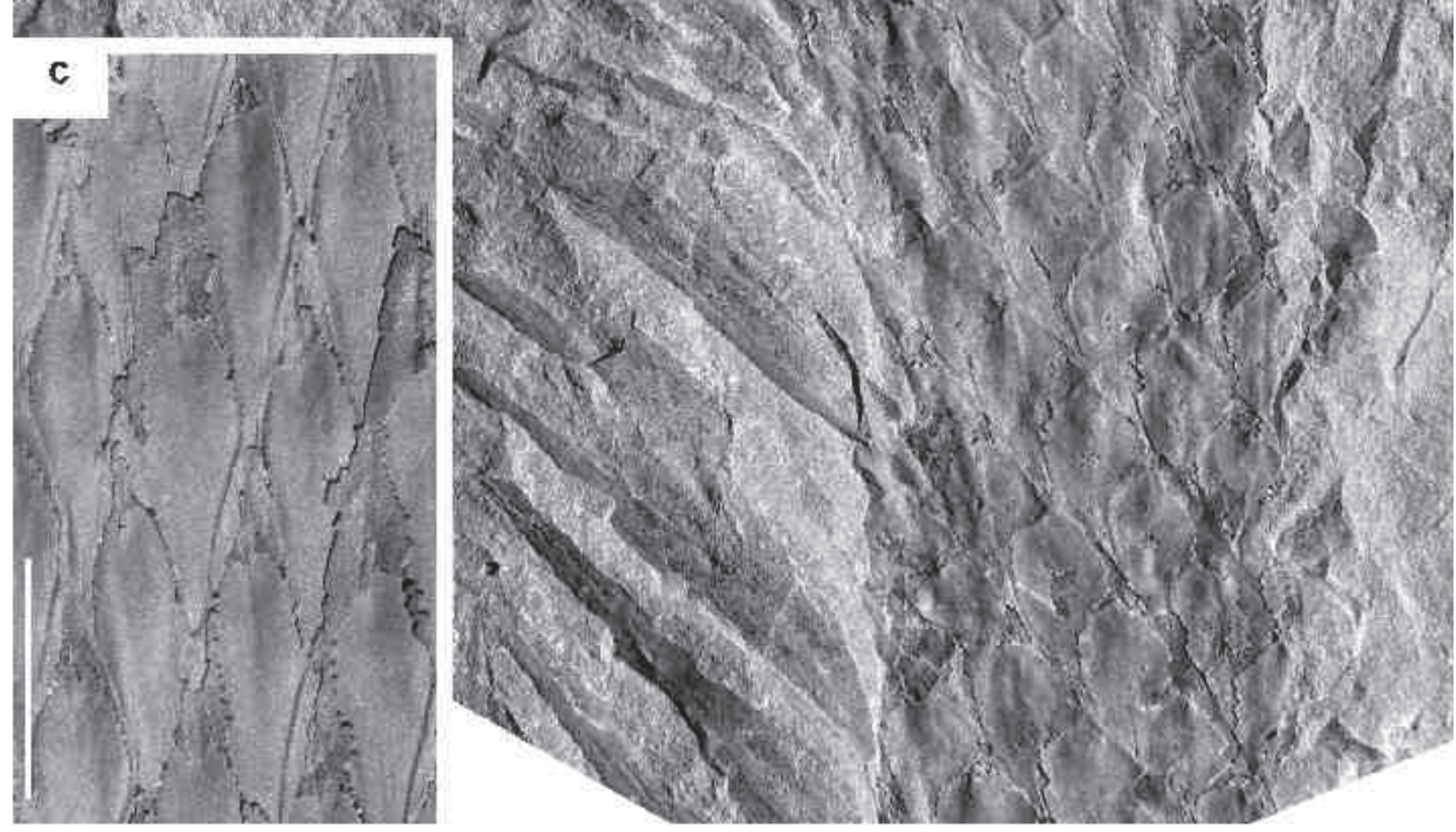

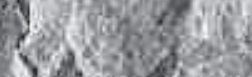

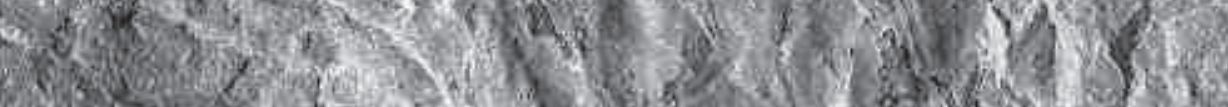

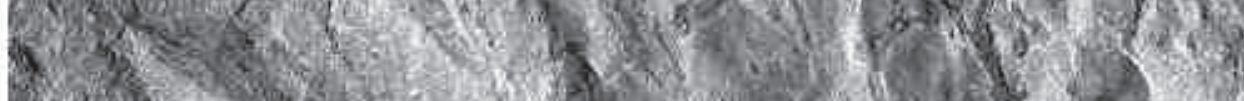
(1) (3)

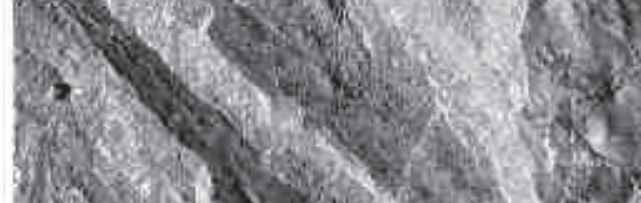

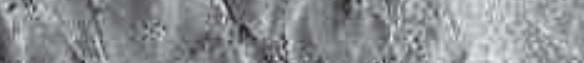
1. $x$ (1)

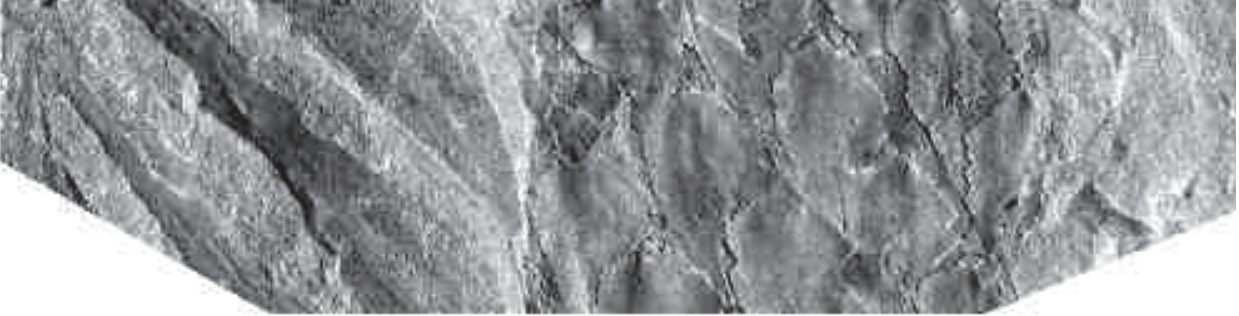


1994 Lepidodendron lycopodioides, Cleal \& Thomas, $\mathrm{p}$. 103, pl. 4, fig. 4; pl. 15, fig. 4 (leafy twig); text-fig. 48E.

p 1994 Lepidodendron ophiurus, Cleal \& Thomas, p. 103, pl. 6, fig. 5; non text-fig. 48C (= "Lepidodendron" ophiurus).

v 1995 Ulodendron acutum, Álvarez-Vázquez, p. 218221, lám. 73, figs 1-2; lám. 74, figs 1, 2; lám. 75, figs 1, 2 (together with Flemingites russelianus); lám. 76, figs 1,6 .

v 1995 Lepidodendron acutum, Wagner \& ÁlvarezVázquez, p. 161, 168.

1996 ?Tomiodendron, Brousmiche Delcambre et al., p. 88,104 , pl. 6, figs 4-5a.

1996 Lepidodendron lycopodioides, Brousmiche Delcambre et al., p. 105, pl. 6, fig. 7 .

? 1996 Lycopodites, Cross et al., p. 478, fig. 23-30.2 (leafy twigs with long leaves that cannot be judged with certainty from the illustration).

? 1997 Lepidodendraceae sp., Brousmiche Delcambre et al., p. 173, pl. I, fig. 2 (poorly preserved).

1997 Lepidodendron acutum, Kvaček \& Straková, p. 28, 34, 100, 126, 130, pl. 2, figs 3, 4 (holotype of Bergeria acuta); pl. 4, fig. 3 (holotype of Bergeria angulata); pl. 33, fig. 5 (holotype of Bergeria marginata); pl. 45, fig. 4 (holotype of Bergeria quadrata); pl. 46, fig. 4 (holotype of Bergeria rhombica).

p 1997 Lepidodendron ophiurus, Migier, p. 261, pl. I, fig. 13; non pl. I, fig. 10 (same as Migier, 1982, Tab. I, fig. 6).

1997 Lepidodendron sp., Migier, pl. I, fig. 19 (together with strobilus).

1998 Lépidodendrale incertae sedis, Brousmiche Delcambre et al., p. 91, 116, pl. 1, figs 3-3a.

v 1999 Ulodendron acutum, Álvarez-Vázquez, figs 2, 4 (name only).

v 2001 Ulodendron acutum, Wagner, p. 95.

? 2003 Lepidodendron species (cf. L. lycopodioides), van Amerom \& Kabon, p. 535, Taf. 4, fig. 1.

? 2007 Lepidodendron acutum, Opluštil et al., pl. VII, fig. 2 (leafy twig difficult to judge from the illustration at reduced size). p 2008 Lepidodendron lycopodioides, van der Veldt, p. 63, fig. 3; non fig. 4 (= "Lepidodendron" ophiurus).

2009 Lepidodendron acutum-L. simile type branches, Opluštil \& Bek, p. 217, fig. 6A (leafy branches associated with strobilus), fig. 6C.

? 2009a "Lepidodendron" simile, Opluštil et al., p. 244-245, 252, pl. I, figs 1-6 (difficult to judge from the illustration).

2009b Lepidodendron simile, Opluštil et al., p. 729, 731, 735, 736, 744, fig. 7C (leafy twigs with long leaves), fig. 7D.

v 2010 Ulodendron acutum, Wagner \& ÁlvarezVázquez, p. 257, 262, 264, 266, 270, 307, pl. XI, fig. 2 (here Figs 5a-5b).

2011 Lepidodendron acutum, Pšenička \& Opluštil, p. 65 , pl. I, figs $1-3,5,7$.

? 2011 Lepidodendron simile, Pšenička \& Opluštil, p. 65 , pl. I, figs 4, 6 (difficult to judge from the illustration).

? 2012 Lepidodendron cf. acutum, Pšenička et al., p. 41, pl. V, fig. 4 (difficult to judge from the illustration).

$\S$ v 2014 Bergeria dilatata, Álvarez-Vázquez \& Wagner, p. 201-204, fig. 13 (copy, at reduced size, of Lindley Hutton's drawing); figs 14a-14h; figs 16g-16h (leafy twigs).

2016 Bergeria dilatata, Opluštil et al., figs 10G, 11D.

2017 Bergeria dilatata, Opluštil et al., p. 268, 274, pl. I, fig. 3 .

? 2017 Bergeria sp., Pšenička et al., p. 16-17, pl. 1, fig. 2 (leafy twig - cannot be judged properly from the illustration).

2017 Synchysidendron, DiMichele et al., p. 5, fig. 4B (Synchysidendron, as described by DiMichele \& Bateman, 1992, possesses differentiated leaf scars and a marked lower keel with distinct plications; none of these characteristics are visible in the specimens figured by DiMichele et al.).

N.B. System of annotations as follows: * Protologue; $\S$ first publication of currently accepted combination; ? references to doubtful specimens due to poor presevation or illustration; $\mathrm{p}$ pars: only part of the specimens published belong to the species; $\mathrm{v}$ vide: the authors have seen the

Figure 7. Bergeria dilatata (Lindey \& Hutton) Álvarez-Vázquez \& Wagner. a) PBE11001 (x 3). Enlargement of lower part of specimen in Fig. 6. Origin: same as for Fig. 6 (locality 10257). b) PBE09352 (x 3). Origin: borehole SBA-92-33, at 29,50 m depth (locality 8203); lower Bolsovian. c) PBE09508 (x 3). Detail of smooth, obovate cushions, with acute apices and bases. Origin: borehole SEP-10, at 39,10 m depth (locality 6738); lower Bolsovian. Scale bars $=1$ cm. Repository: Centro Paleobotánico, Real Jardín Botánico de Córdoba, Spain. 


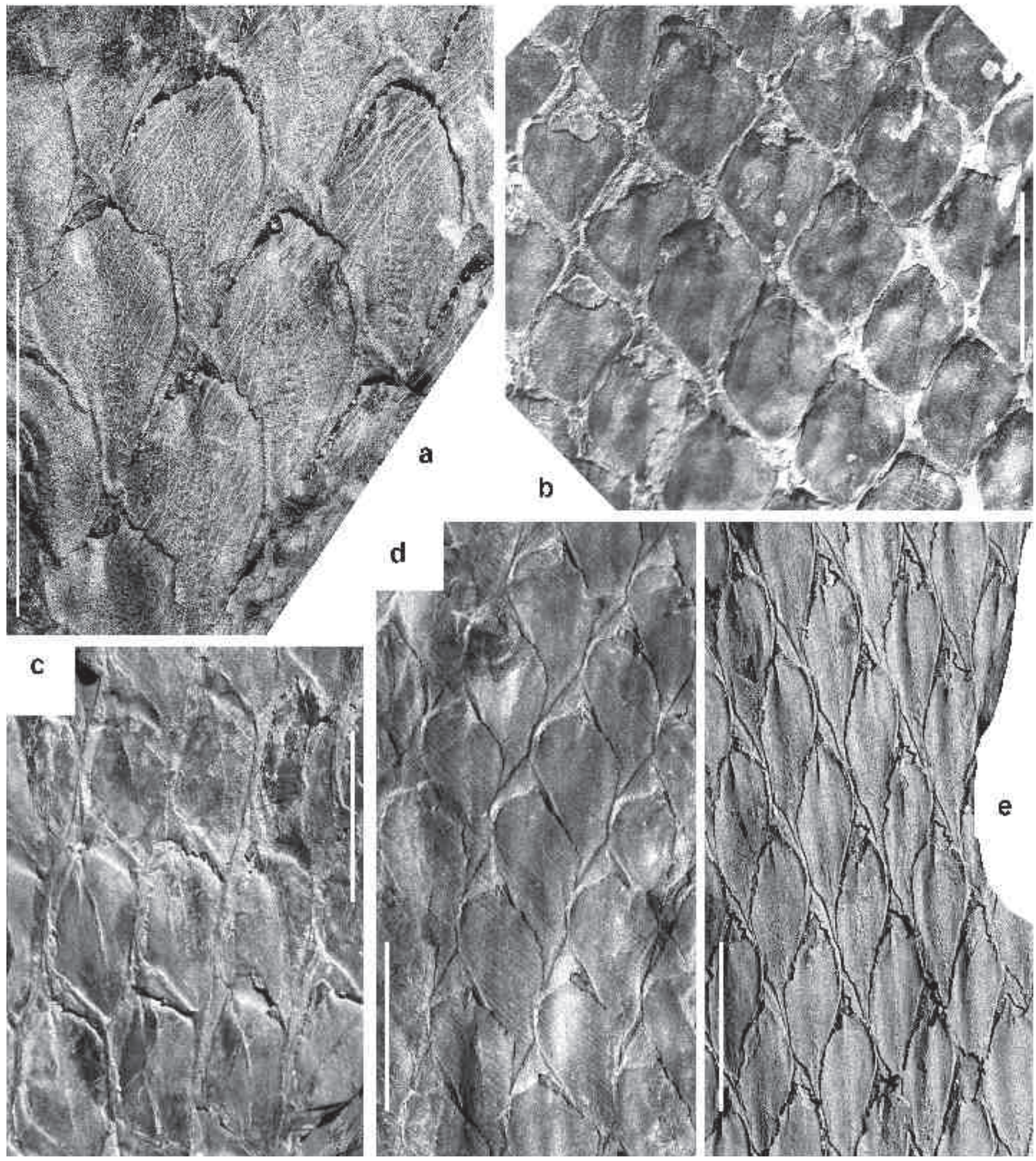

Figure 8. Bergeria dilatata (Lindey \& Hutton) Álvarez-Vázquez \& Wagner. a) PBE11002. Obovate leaf cushions showing barely distinct keels crossed with short wrinkles. Origin: Corta Ballesta; 1,10 m above Capa (Seam) 9 (locality 10344); lower Bolsovian. b) PBE09573. Rhomboidal leaf cushions separated by narrow grooves. Origin: borehole SB-11, at 60,25 m depth (locality 7266); lower Bolsovian. c) PBE11005. Origin: same as Fig. 8a (locality 10344). d) PBE09510. Note the slightly inflected bases. Origin: borehole SEP-5bis, at 324,70 m depth (locality 5632); lower Bolsovian. e) PBE11003. Note the similarity with one of the types of Lepidodendron lanceolatum (Lesquereux, 1879, pl. LXIII, figs 5-5a). Origin: same as Figs 8a and $8 \mathrm{c}$ (locality 10344). Scale bars $=1 \mathrm{~cm}$. Repository: Centro Paleobotánico, Real Jardín Botánico de Córdoba, Spain. 
specimen(s); k: reference includes cuticular evidence; acc.: according to. We have tried to compose a complete list of synonymy, ignoring only mere textual citations unless these have been reviewed by the present authors.

Material and preservation. An extensive collection from 78 localities in the Peñarroya-Belmez-Espiel Coalfield comprises a total of 586 specimens; these include a few large stem fragments (up to $30 \mathrm{~cm}$ long and $8 \mathrm{~cm}$ wide) as well as numerous medium-sized leafy branches (the most frequent: up to $25 \mathrm{~cm}$ long and $2 \mathrm{~cm}$ wide), and small leafy twigs. Several tens of them are one to four times dichotomized at an acute angle. Specimens are preserved as adpressions on siltstones. They are identified by engraved locality numbers and a catalogue number preceded by PBE (identifying the coalfield). All material was photographed dry and with natural lighting.

Lindley \& Hutton's material consists of three specimens that originated from the roof of the Low Main coal seam, Felling Colliery, Newcastle-upon-Tyne, England. Catalogue numbers are HM G02.16 (Lindley \& Hutton, 1831, pl. 7, fig. 2 - holotype of Lepidodendron dilatatum), HM G02.17 (Lindley \& Hutton, 1834, pl. 118) and HM G02.20 (Lindley \& Hutton, 1834, pl. 113).

Age. Late Langsettian/early Duckmantian and early Bolsovian for the material from Spain. Lindley \& Hutton specimens originated from lower Duckmantian strata (see Ramsbottom et al., 1978).

Repository. Spanish material is stored in the Fossil Plant Collection of the Centro Paleobotánico, Real Jardín Botánico de Córdoba, Córdoba, Spain. Lindley \& Hutton's specimens are in the collections of the Great North Museum-Hancock, Newcastle-upon-Tyne, England.

Diagnosis. Lycopsid stems, branches and leafy twigs covered with spirally arranged leaf cushions. Branches isotomously forked at acute angles. Leaf cushions contiguous or separated by narrow grooves; smooth, obovate to rhomboidal, longer than broad, broadest in the upper part of cushion; straight or slightly inflected acuminate base, acute apex and rounded lateral angles. Parichnos markings absent. Keel absent or very faintly marked. Long, grass-like leaves, single-veined, inserted at acute angles. A very small, irregular scar marks the position of leaf base at the top of the cushion where leaves have been detached.

Description. Stem and branch fragments up to $55 \mathrm{~cm}$ long and $11 \mathrm{~cm}$ wide, straight, covered with closely spaced, helicoidally disposed leaf cushions; these are longer than broad, contiguous in the smaller branches and separated by very narrow grooves (less than $1.2 \mathrm{~mm}$ broad) in the larger ones. Distal, leafy stems up to $38 \mathrm{~cm}$ long and 2 $\mathrm{cm}$ wide, branching isotomously at acute angles (30-60 - Figs 3, 4, 6). Gradual change in size and shape of leaf cushions below and above dichotomies.

Neither the specimens in the Peñarroya-Belmez-Espiel collection nor those of Lindley \& Hutton show ulodendroid branch scars, but the two syntypes of the synonymous Lepidodendron landsburgii Kidston (1893, pl. III, figs 9, 10; also Crookall, 1964, pl. LXIII, figs 1, 2) show large, oval scars. Kidston's specimens are larger than those described here; although only partly figured, the specimen in his pl. III, fig. 9 measures $28.5 \mathrm{~cm}$ long and $25 \mathrm{~cm}$ wide and possesses two branch scars separated by $18.5 \mathrm{~cm}$ (Kidston, 1893, p. 338). This specimen also shows more distant leaf cushions, separated by slightly wider grooves (up to $2 \mathrm{~mm}$ ) ornamented with flexuous, irregular markings.

Leaf cushions slightly asymmetrical vertically and horizontally, flat and smooth in the medium-sized and smaller branches (e.g. Figs 6, 7a, 7b, 8d), more convex, smooth (Figs 5a, 5b, 9a) or with a very faintly marked, occasionally ornamented keel (Fig. 8a), in the larger ones. Rhomboidal (Figs 2, 8b, 8c, 9a, 9b) to obovate (e.g. Figs $5 \mathrm{a}, 5 \mathrm{~b}, 5 \mathrm{c}, 8 \mathrm{~d}, 8 \mathrm{e})$, broadest in the upper third or upper half of cushion, straight or very slightly inflected in opposite directions; apex acute in obovate cushions (e.g. Figs 7c, $8 \mathrm{~d}, 8 \mathrm{e}$ ), broadly acute to obtuse in rhomboidal ones (Figs 2, 9a, 9b); base acute (Figs 7c, 8d, 8e) or truncate due to slight overlapping between rows of leaf cushions (Figs 9a, 9b); lateral angles more or less rounded in all cases. Dimensions: 3-18 $\mathrm{mm}$ long and 1.5-8 $\mathrm{mm}$ broad; length/ breadth ratio $=2$ to 6 (most commonly $2.5-3.5)$. (True) leaf scar absent; when leaves are detached, a small, irregular scar is seen to mark the position of the leaf base at the top of the leaf cushion (e.g. Figs 7b, 8e, 9b). There is no evidence of either a ligule pit or parichnos markings.

Long, single-veined, straight, entire, grass-like leaves (Figs 2, 5a, 5b, 6, 7a, 10, 10b); they are slightly curved near the base and inserted in total continuity in the uppermost part of leaf cushion at narrow, acute angles (20-40 $)$, tapering gradually to an acuminate apex. Dimensions: up to $80 \mathrm{~mm}$ long and $2-5 \mathrm{~mm}$ width at the base; leaf length at least five times that of the leaf cushions.

Comparisons. The presence of rhomboidal, smooth, longer than broad leaf cushions with small, irregular (false) leaf scars at the top of the cushion, and (when preserved) the long, grass-like leaves make Bergeria dilatata a distinctive taxon.

Bergeria worthenii (Lesquereux) Álvarez-Vázquez \& Wagner (Lesquereux, 1866, pl. XLIV, figs 4, 5; Janssen, 1940, pl. I, figs 3, 4; Bell, 1944, pl. XLVII, figs 2, 4; pl. L, fig. 2; pl. LIV, fig. 4; Janssen, 1957, fig. 19; Laveine et al., 2003, pl. VII, figs 1, 2; Wagner \& Álvarez-Vázquez, 2010, pl. XI, figs 3-3a; Álvarez-Vázquez \& Wagner, 2014, figs $15 \mathrm{a}-15 \mathrm{e}$ ) shows smaller, fusiform to obovate leaf cushions with a markedly convex surface. In addition, the narrow, 

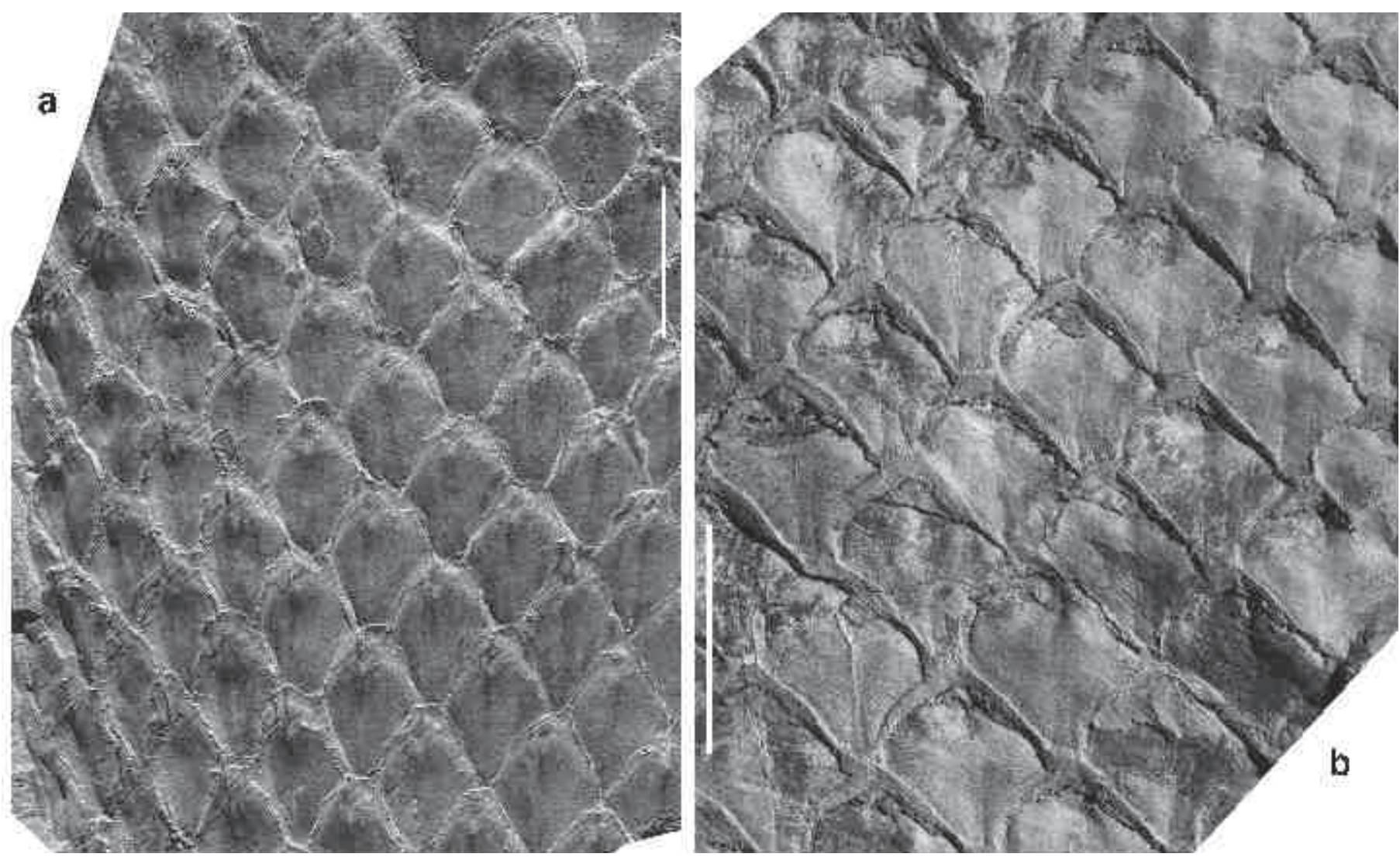

Figure 9. Bergeria dilatata (Lindey \& Hutton) Álvarez-Vázquez \& Wagner. a) PBE09458 (x 2). Stem fragment with rhomboidal leaf cushions showing obtuse apex and truncate base. Origin: Pozo (shaft) Espiel, Rampa 4W, $3^{\text {a }}$ Traviesa, $6 \mathrm{~m}$ below Capa (Seam) Candelaria (locality 6769); lower Bolsovian. b) PBE11004 (x 3). Truncate bases due to overlapping between rows of leaf cushions. Origin: Corta Ballesta; 1,10 m above Capa (Seam) 9 (locality 10344); lower Bolsovian. Scale bars = 1 cm. Repository: Centro Paleobotánico, Real Jardín Botánico de Córdoba, Spain.

irregular, transversally oval to punctiform (false) leaf scar is placed in the upper third of the leaf cushion and occupies almost the entire cushion width. There is no keel; the field area above and below the leaf scar is almost totally covered by relatively coarse, transverse, discontinuous wrinkles. Bergeria worthenii ranges through the entire Westphalian.

The Langsettian species Bergeria wingfieldense (Thomas \& Seyfullah, 2015) comb. nov. [Basionym: Lepidodendron wingfieldense Thomas \& Seyfullah, 2015, Palaeontographica, Abt. B, 292 (1-3), p. 31, text-figs 4A5D, text-fig. 5E (strobilii), text-fig. 5F (megaspore), textfigs 6A-6F (cuticles)] is characterized by rhomboidal, shorter and more equidimensional cushions, with a length/ breadth ratio of $\sim 1.2$. Since no proper leaf scar is seen, we exclude this species from Lepidodendron. Thomas \& Seyfullah (2015) only described seven specimens (together with two strobili), which seems insufficient to show the intraspecific variability. However, apart from the size and shape, the well-marked, ornamented keel with short transverse wrinkles allows Bergeria wingfieldense to be easily distinguished from Bergeria dilatata. Associated strobili possess Lagenoisporites-type megaspores and were compared by Thomas \& Seyfullah (2015) with Flemingites olryi (Zeiller) Brack-Hanes \& Thomas.

Also similar is the Westphalian species Bergeria lycopodioides (Sternberg, 1821) comb. nov. [Basionym: Lepidodendron lycopodioides Sternberg, 1821, Versuch einer geognostisch-botanischer Darstellung der Flora der Vorwelt, p. 26, Taf. XVI, figs 1, 2, 4 - refigured by Kvaček \& Straková, 1997, pl. 32, and as "Lepidodendron" selaginoides by Němejc, 1947, Tab. II, fig. 6, Tab. III, fig. 2] and its synonym "Lepidodendron" selaginoides (Sternberg, 1821, Taf. XVI, fig. 3, Taf. XVII, fig. 1; copy of the original drawing in Cleal et al., 2005, p. 47, fig. 5 (1); and refigured by Němejc, 1947, Tab. III, figs 1, 3; Kvaček \& Straková, 1997, pl. 50, figs 1, 3; and Opluštil et al., 2007, pl. V, fig. 9). Bergeria lycopodioides possesses contiguous, fusiform, elongate leaf cushions with upper and lower ends that are slightly inflected in opposite directions. Leaves are apparently linear, and much shorter than in Bergeria dilatata. Opluštil et al. (2009b, fig. 7A) illustrated a large specimen (more than $70 \mathrm{~cm}$ long) showing a wide stem fragment as well as numerous medium-sized branches and small twigs, several times dichotomized at acute angles. 
The detail of leaf cushions (op. cit., fig. 7B) shows no proper leaf scars, but a small, irregular, punctiform scar in the upper third of the leaf cushions. A well-marked keel, crossed by relatively coarse, transverse wrinkles (similar to those seen in Bergeria worthenii) is seen in the field below the false leaf scars. There is no parichnos. As in the case of Lepidodendron wingfieldense, Lepidodendron lycopodioides must be excluded from Lepidodendron.

Comparison can also be made with "Lepidodendron" ophiurus (Brongniart) Brongniart (Brongniart, 1822, pl. IV, figs 1a-1b - drawings reproduced in Crookall, 1964, textfig. 93; Thomas \& Seyfullah, 2015, text-figs 1A, 1B; textfigs 2A-D), but this species shows much smaller, fusiform leaf cushions, with acute upper and lower angles and rounded laterals, showing a well-marked, unornamented keel. There is no parichnos. Leaves are much shorter than those of Bergeria dilatata and are attached at about one third down the leaf cushion at near right angles, curving upwards more or less parallel to the stem. Although this species should be excluded from Lepidodendron, the presence of small, rhomboidal leaf scars when leaves are not preserved, confirms that it can not be included in Bergeria.

The lower Westphalian species "Lepidodendron" belgicum (Kidston, 1911, pl. XVII, figs 1-3) shows similar, contiguous, longer than broad, obovate to rhomboidal leaf cushions. Poorly-defined, relatively short leaves are observed in some areas of the type material (three larger stems and two twigs in the same slab), but leaf cushions possess a well-defined, rhomboidal leaf scar located in the upper part. This excludes "Lepidodendron" belgicum from Bergeria. Additionally, there is a distinctive keel crossed by well-marked, short, transverse wrinkles in the lower field; a large branch scar is seen in one of the specimens. Although a revision of the original material would be necessary, Kidston's specimens do not show parichnos, so this species should also be excluded from Lepidodendron.

Small branches with attached leaves of Bergeria dilatata are distinguished from those of Bothrodendron minutifolium (Boulay) Zeiller (Boulay, 1876, pl. III, figs 1-1bis; Zeiller, 1879, p. 117-118; Zeiller, 1886, pl. LXXIV, figs $2-4 C$ ) by the presence, in the latter, of more tightly adpressed, shorter (always less than $10 \mathrm{~mm}$ long), linearlanceolate leaves with a sharply pointed apex. Moreover, Bothrodendron minutifolium leaves are attached to the middle part of the very small, rhomboidal-elongated leaf cushions, not at the top as in Bergeria dilatata.

Genus Flemingites Carruthers, 1865 emend. BrackHanes \& Thomas, 1983

Type-species Flemingites gracilis Carruthers, 1865

Remarks. Flemingitacean cones are characterized by the occurrence of megasporangia in the basal part and microsporangia near the apex. Brack-Hanes \& Thomas (1983) restricted Lepidostrobus to monosporangiate cones with cingulizonate and cingulate Lycospora type microspores, and Flemingites to bisporangiate strobili with megaspores of the Lagenicula and Lagenoisporites types and microspores of Microspinosporites type. Not all flemingitacean cones yield both in situ micro- and megaspores. It is not unusual to find flemingitacean specimens (preserved as fragments) that yield only microor megaspores, but if the in situ spores are identified to these characteristic types, this is strong confirmation of the generic classification of the strobilus (see Bek, 2017). In situ flemingitacean microspores have been referred to Lycospora (Schopf, Wilson \& Bentall) Potonié \& Kremp, mostly to the dispersed miospore species Lycospora orbicula (Potonié \& Kremp) Smith \& Butterworth or Lycospora granulata Kosanke (Bek, 2017). These microspores were originally interpreted as cingulate (Potonié \& Kremp, 1955; Smith \& Butterworth, 1967), but Bek (2013) re-defined these spores as monopseudosaccate, excluding them from Lycospora, and proposed a new genus, Microspinosporites. Flemingitacean cone species with megaspores of the Lagenoisporites type (see Bek, 2017) are significantly more numerous than those that produced Lagenicula.

This group of flemingitacean cones includes not only true Flemingites but also two uppermost Devonian Sublepidodendron species (Bek, 2017). The genus Thomasostrobus Opluštil et al. appears to represent a special position among Pennsylvanian bisporangiate cones, being bisporangiate but probably close to some sigillarians that are monosporangiate.

\section{Flemingites russelianus (Binney, 1871) Brack-Hanes \& Thomas, 1983 \\ (Figs 11a-11j)}

* p 1871 Lepidostrobus Russelianus Binney, p. 51, pl. IX, figs 1, 1a; non pl. IX, figs 2, 2a (Lepidostrobus dubius Binney acc. to Chaloner, 1953).

* 1871 Lepidostrobus Hibbertianus Binney, p. 55, pl. X, figs 2-2b (acc. to Chaloner, 1953).

1928 Lepidostrobus sp., Šusta, Taf. VIII, Abb. 2 (associated with small branches that have been included here in the synonymy of Bergeria dilatata).

1937 Lepidostrobus cf. Goodei, Jongmans, 397, pl. 15, fig. 25.

1949 Lepidostrobus sp., Arnold, p. 172-173, pl. VII, figs 1, 3, 4 (acc. to Chaloner, 1953).

? 1952 Ulostrobus Goodei, Stockmans \& Willière, pl. E, fig. 1 (in association with Ulodendron goodei, here included in Bergeria dilatata); pl. F, figs 1, 1a, fig. 2 (together with Ulodendron goodei), figs 3-5a. 

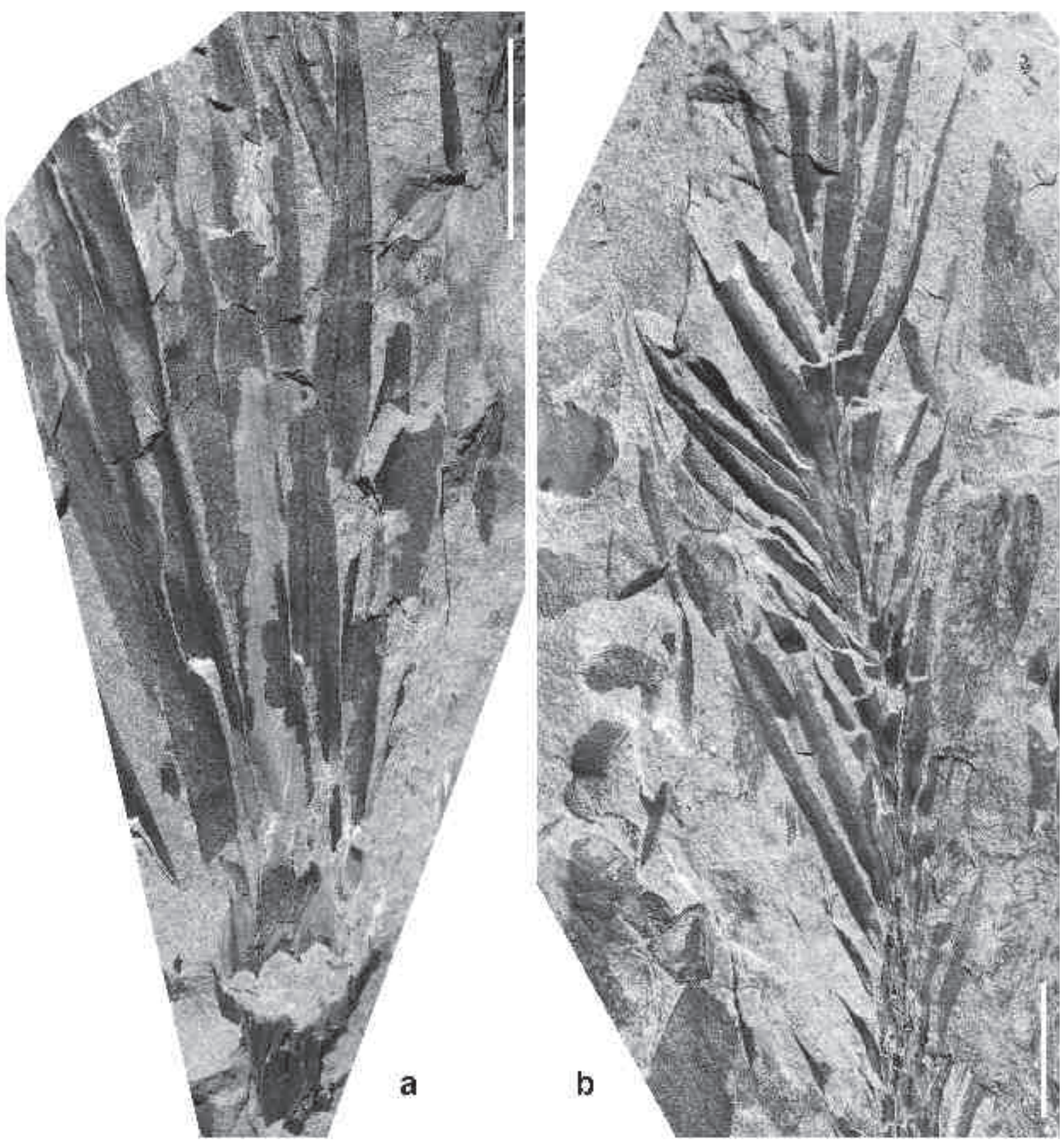

Figure 10. Bergeria dilatata (Lindey \& Hutton) Álvarez-Vázquez \& Wagner. a) PBE11007 (x 3). Detail of the long, entire, single-veined leaves. Origin: Corta Ballesta; 1,10 m above Capa (Seam) 9 (locality 10344); lower Bolsovian. b) PBE11006 (x 2). Leafy twig. Origin: same as Fig. 10a (locality 10344). Scale bars $=1 \mathrm{~cm}$. Repository: Centro Paleobotánico, Real Jardín Botánico de Córdoba, Spain.

1953 Lepidostrobus russelianus, Chaloner, p. 277 (emended diagnosis), text-figs 13-16 (megaspores), 17A-E (microspores).

* 1966 Lepidostrobus russelianus, Crookall, p. 500, text-fig. 147 (copy of Binney's figures).

$\S 1983$ Flemingites russelianus, Brack-Hanes \& Thomas, p. 132.

v 1995 Flemingites russelianus, Álvarez-Vázquez, p. 222, lám. 74, fig. 3; lám. 75, fig. 2; lám. 77, figs 1-3.
1998 Lepidostrobus sp. 1, Brousmiche Delcambre et al., p. 92, 117, pl. 2, figs 1-1a.

v 1999 Flemingites russelianus, Álvarez-Vázquez, fig. 4 (name only).

v 2001 Flemingites russelianus, Wagner, p. 98.

p 2009 Flemingites $\mathrm{cf}$. russelianus, Opluštil \& Bek, p. 216-218, fig. 6A (together with Bergeria dilatata), fig. 6B; figs 6D-6G (spores). 
v 2010 Flemingites russelianus, Wagner \& ÁlvarezVázquez, p. 266.

Material. Twenty-three specimens found in association with stem and leafy twigs of Bergeria dilatata. Two specimens, from localities 6789 (Pozo Espiel) and 10251 (Corta Ballesta), were macerated for palynology. Megaspores were macerated in nitric acid (24 hours), treated in potassium hydroxide (20 minutes) and washed in distilled water. Microspores and dispersed miospores and pollen were macerated in hydrofluoric acid (5 days), nitric acid (24 hours) and treated in potassium hydroxide (20 minutes). Descriptive terms for the spores follow the latest edition of the Glossary of Pollen and Spore Terminology (Punt et al., 2007). Megaspores are classified based on Dybová-Jachowicz et al. (1979, 1982, 1987a, 1987b). Microspores are classified according to the system of dispersed spores suggested by Potonié \& Kremp $(1954,1955)$, Smith \& Butterworth (1967) and Bek (2013). In situ spores were compared directly with the original diagnoses, type specimens, description, and illustrations of dispersed spore species. Species determinations are based solely on the original diagnoses, not on the interpretations of subsequent authors.

Diagnosis. Strobilus cylindrical, slightly tapering from base to apex; axis wide, with perpendicular or nearly perpendicular, closely adpressed sporophylls. Megasporangia concentrated in lower part of strobilus and microsporangia in apical part. In situ megaspores of the Lagenoisporites rugosus type and microspores of the Microspinosporites orbiculus type.

Description. Strobilus cylindrical, elongate, slightly tapering from base to apex, at least $120 \mathrm{~mm}$ long (incomplete) and $18-20 \mathrm{~mm}$ wide. Axis 2-2.5 mm wide, with perpendicular or nearly perpendicular sporophylls arranged in pseudowhorls and separated by c. $1 \mathrm{~mm}$. Sporophyll pedicel up to $15 \mathrm{~mm}$ long, inserted perpendicular to the axis; upper part of the sporophyll closely adpressed and abruptly ascending parallel to the axis, with a relatively thick, subtriangular and singleveined lamina; margins entire. Sporophyll dimensions: up to $12 \mathrm{~mm}$ long and $25 \mathrm{~mm}$ width.

Megasporangia concentrated in the lower part of strobilus and microsporangia in the apical part. Sporangia 5-7 mm long (measured radially) and $1.5-2 \mathrm{~mm}$ wide (parallel to the axis). Trilete megaspores flattened laterally, more or less oval; those in proximal-distal direction, round or oval (Figs 11b, 11f-j). Apical prominence distinct. Arcuate ridges distinctly marked in well-preserved specimens. Megaspores 550 (883) $1210 \mu \mathrm{m}$ in diameter, gula 240 (310) $400 \mu \mathrm{m}$ high and 180 (226) $300 \mu \mathrm{m}$ wide (Figs 11b, 11g, 11i). Exine punctate to rugose, $10-20 \mu \mathrm{m}$ thick. Many megaspores have small globules called ubisch bodies (or their imprints) on the surface (Fig. 11h). Trilete pseudosaccate miospores, 23-36 $\mu \mathrm{m}$ in diameter (Figs 11a, 11c-e). Amb circular to oval, margin microspinate. Laesurae simple, equal to the radius of the inner body, sometimes indistinct. Distal surface microspinate, proximal surface microspinate except for laevigate contact area. In situ megaspores of the Lagenoisporites rugosus type and microspores of the Microspinosporites orbiculus type.

Comparisons. Although containing both megaspores of the Lagenoisporites type and microspores of Microspinosporites type, cones of the lower to middle Westphalian species Flemingites olryi (Zeiller) BrackHanes \& Thomas are smaller (less than $12 \mathrm{~mm}$ wide) and have a higher length/breadth ratio than those of Flemingites russelianus.

Cones of Lepidostrobus ornatus Brongniart are also large, long and cylindrical. However, they are microsporangiate, with microspores of the Lycospora type.

The lower Westphalian, monosporangiate cones of Lepidostrobus spinosus Kidston are smaller, oval, with an obtuse apex. Microspores are of the Lycospora type (Opluštil \& Bek, 2009).

Remarks. There are at least 17 Paleozoic flemingitacean strobilus form-species that are recorded to have produced miospores of the Microspinosporites type (Bek, 2017) and 16 species that yielded megaspores of the Lagenoisporites rugosus type. All these in situ micro- and megaspores are closely similar and differ only slightly in diameter. All other morphological features are identical, i.e. sculpture, cingulum, gula, rays of trilete mark, etc. As such, it is not possible to distinguish parent plants of Microspinosporites orbiculus and Lagenoisporites rugosus based solely on their in situ spores. Comparisons can be made based on the morphology/anatomy of the cones and the whole parent plant, if cones are in connection.

Stratigraphic distribution. Flemingites russelianus has been recorded from Langsettian to Bolsovian strata. The type specimens came from the Blackband Ironstone, Airdrie, Lanarkshire, Scotland, from upper Langsettian strata. Chaloner (1953) noted the association of Flemingites russelianus cones with Lepidodendron acutum (= Bergeria dilatata).

\section{DISPERSED MIOSPORE AND POLLEN ASSEMBLAGE}

A small piece of the matrix $(4 \times 4 \times 1 \mathrm{~cm})$ was macerated to obtain a dispersed miospore and pollen assemblage associated with studied specimens of Bergeria dilatata; based on our knowledge of in situ spores and pollen (e.g. Balme, 1995), this provides insight into the vegetation 

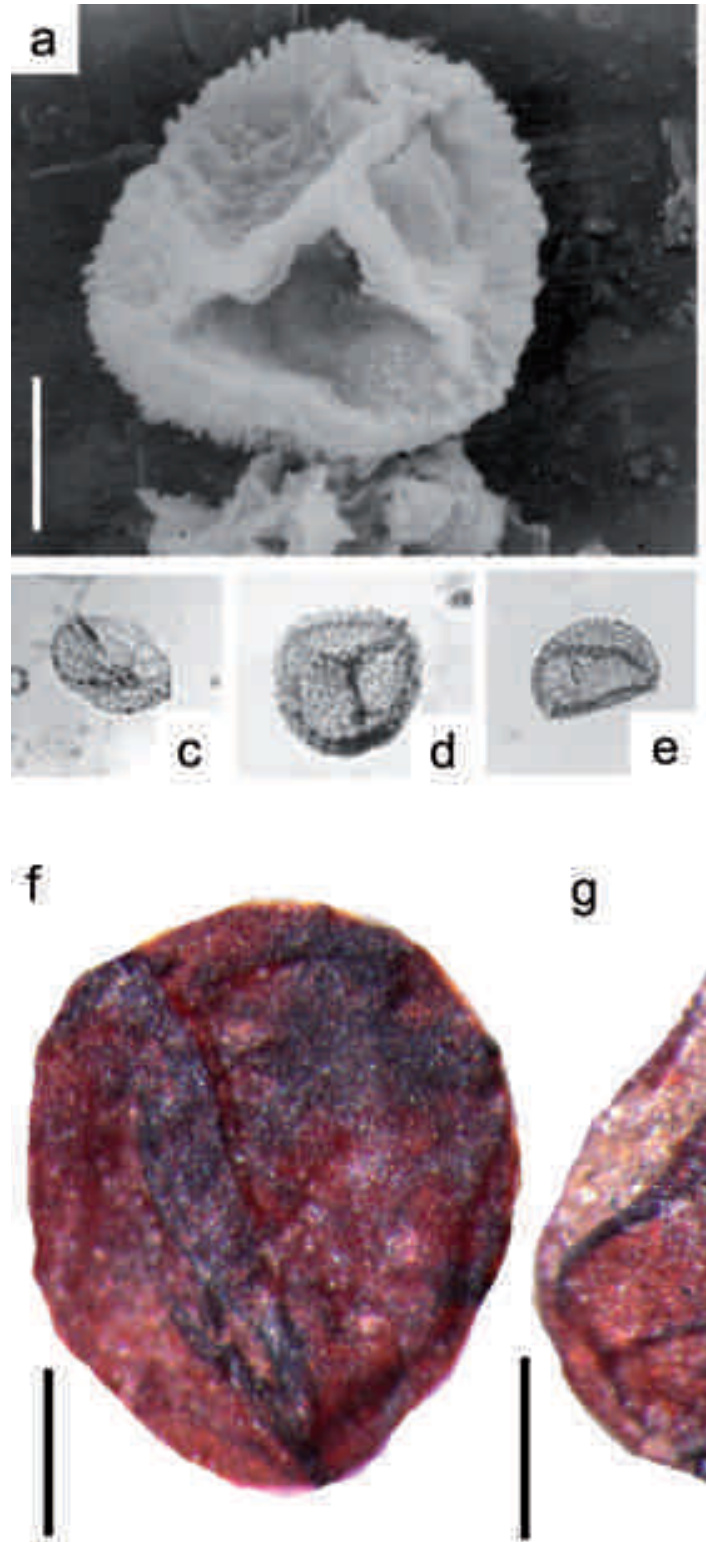

e $1: 2$

b.

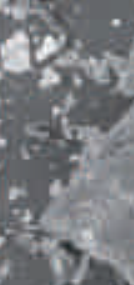

$x=$

ह5? 30 $+6$<smiles>C=CCC(CC)CC(CC)C1CCCCC1</smiles>

\section{.}

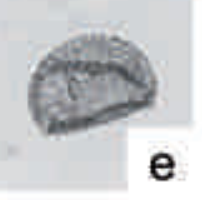

$\therefore$

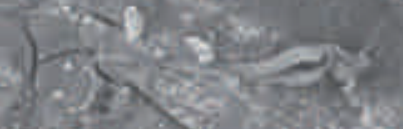

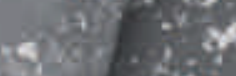

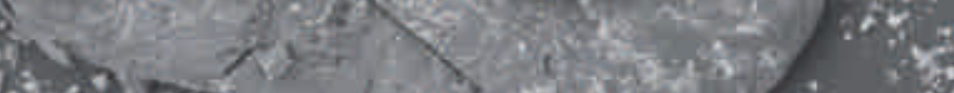

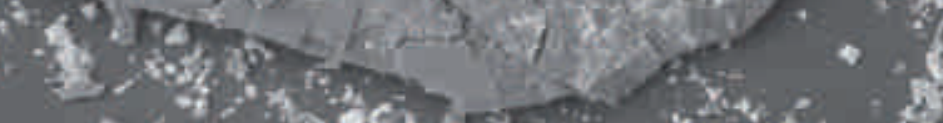

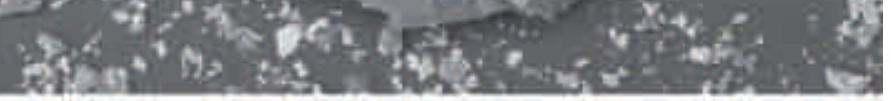

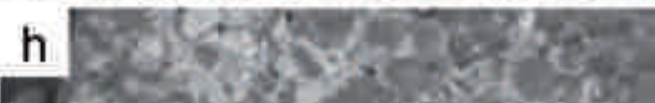

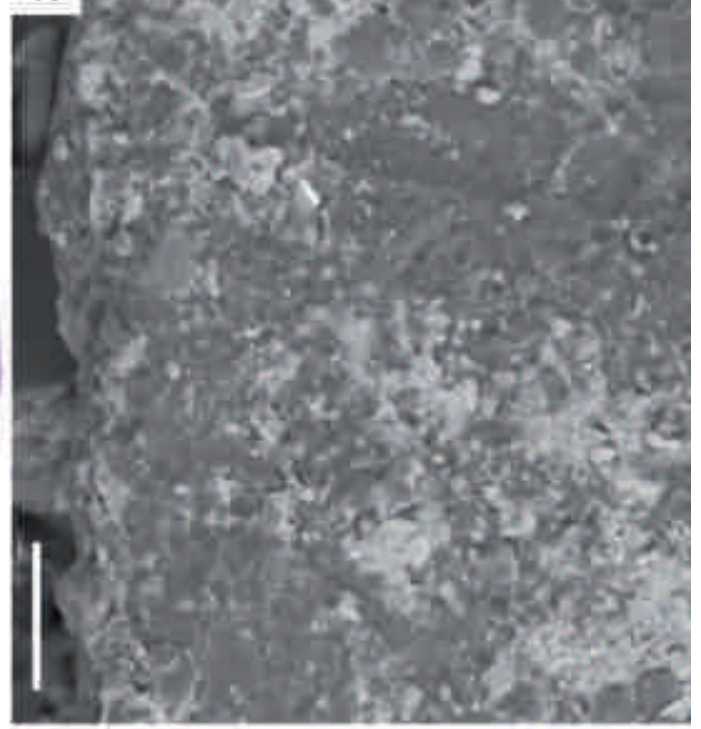

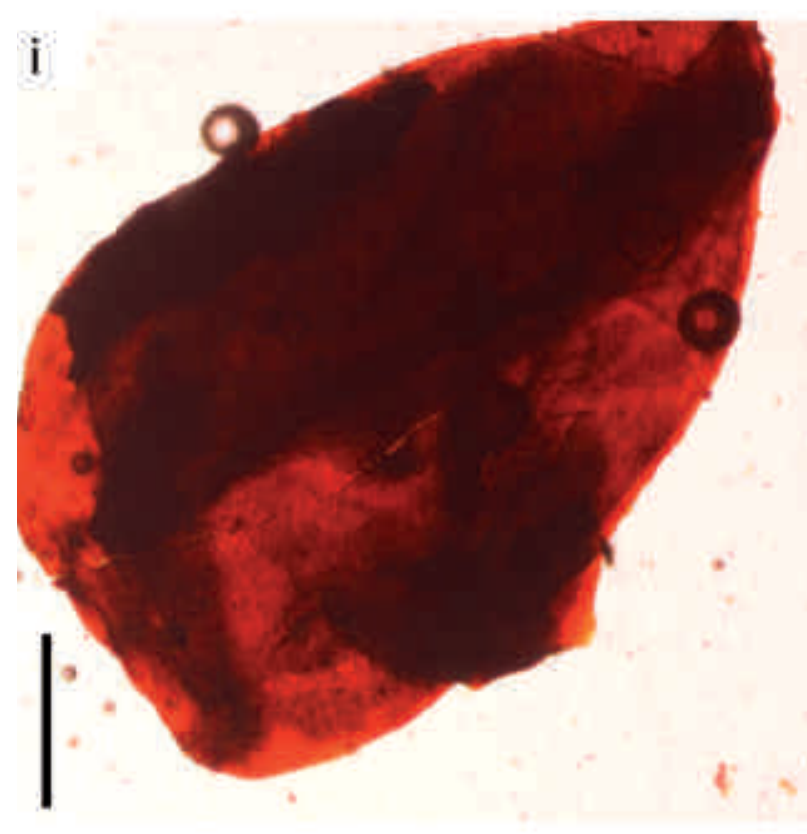

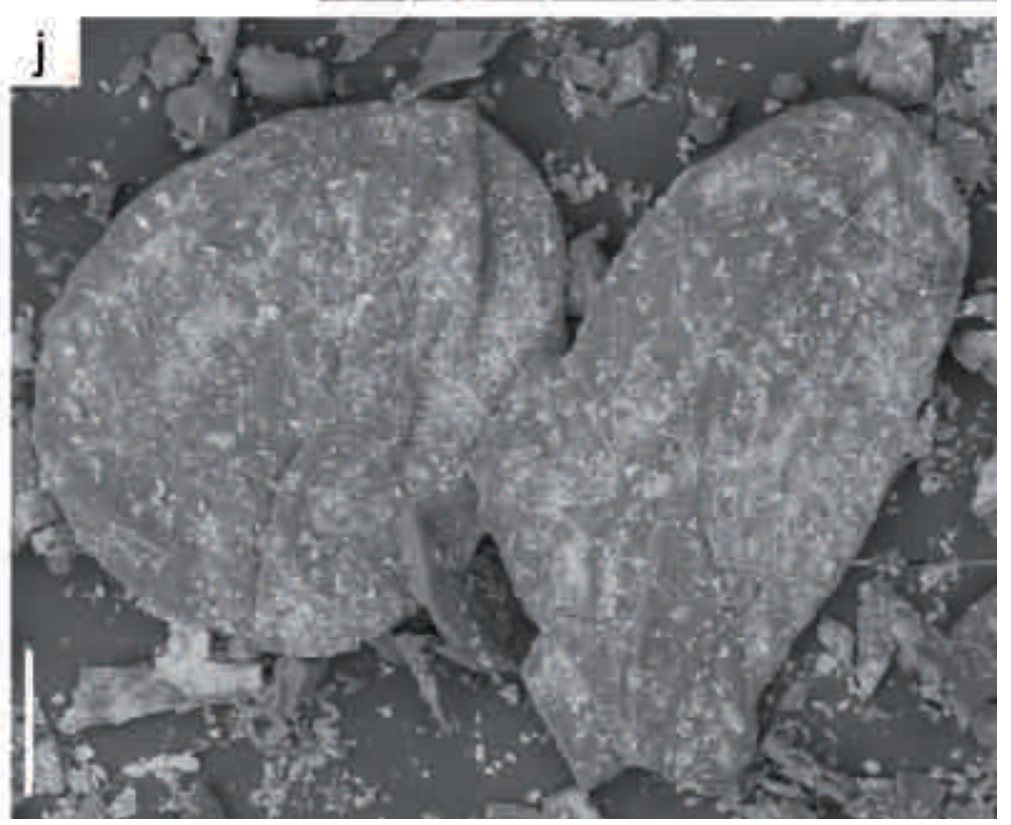


growing in the same general area as Bergeria dilatata. The dispersed miospore and pollen assemblage consists of 37 species belonging to 22 genera. The most abundant were arborescent lycopsids (attributed to Lepidodendron, Lepidophloios and Sigillaria types). They are represented mainly by several species of the genus Lycospora and the flemingitacean microspore genus Microspinosporites, i.e. the same species as macerated in situ from the cones. The second most abundant miospore type is that attributed to marattialean and botryopterid ferns; less abundant miospore types are attributed to zygopterid and gleicheniacean fern taxa. The miospore record of other groups, including herbaceous lycopsids, Calamites, sphenophylls, cordaitaleans, conifers and pteridosperms, is poorly represented.

The predominance of miospores of arborescent lycopsids of the Lepidodendron and Lepidophloios type points to typical peat-forming vegetation of a Pennsylvanian rheotrophic peatland.

\section{HABITAT}

As mentioned above, Bergeria dilatata is the most common species of lycopsid in the Peñarroya-BelmezEspiel Coalfield. It is noted that it never appears in direct association with a coal seam, and that it is frequently associated (28 localities; almost a third of the total localities) with sphenopsids: mainly Calamites suckowii Brongniart and Calamites cistii Brongniart, and less commonly Sphenophyllum cuneifolium (Sternberg) Zeiller, Annularia sp., Asterophyllites sp. and Calamostachys sp. Another frequent association is with pteridosperms: Neuropteris guadiatensis Wagner emend. Álvarez-Vázquez, Laveineopteris tenuifolia (Schlotheim ex Sternberg) Cleal, Shute \& Zodrow, Paripteris linguaefolia Bertrand and Mariopteris muricata (Schlotheim) Zeiller. Less commonly (only at one locality in each case), it is associated with Bothrodendron minutifolium (Boulay) Zeiller, Wagneropteris minima Álvarez-Vázquez \& Cleal, Lonchopteris rugosa
Brongniart, Palmatopteris furcata (Brongniart) Potonié, Senftenbergia plumosa (Artis) Stur, Sturia amoena (Stur) Němejc, Renaultia sp. and Pecopteris sp.

It is further noted that Bergeria dilatata occurs in siltstones above coal seams, forming part of the fining upward sequences of alluvial plain facies (see Fig. 12 containing localities 8203, 10251, 10257 and 10344 in Corta Ballesta, which contributed more than 300 of the 586 specimens assigned to Bergeria dilatata in the Peñarroya-Belmez-Espiel Coalfield). It may be interpreted that, in contrast to other lycopsids, this species inhabited open flooded areas with higher clastic input; in effect, a pioneer species.

It is considered relevant to compare with the interpretation of DiMichele \& Phillips $(1985,1994)$ and Phillips \& DiMichele (1992) of the anatomically preserved Paralycopodites, which was a small (less than $10 \mathrm{~m}$ tall), straight tree with two opposite rows of deciduous lateral branches of relatively small size. Its leaves also remained attached to lateral branches and to the main trunk. Paralycopodites would appear also to have been a pioneer element living in open, clastic, disturbed parts of swamp areas, probably not subjected to long-term flooding.

\section{GEOGRAPHIC AND STRATIGRAPHIC DISTRIBUTION}

The only specimen (holotype) figured by Lindley \& Hutton (1831, pl. 7, fig. 2) as Lepidodendron dilatatum originated from the roof of the Low Main coal seam, Felling Colliery, near Newcastle-upon-Tyne, England, from strata of early Duckmantian age. Lindley \& Hutton's Lepidodendron gracile, and the specimens figured by them as Lepidodendron sternbergii Brongniart, Lepidodendron selaginoides Sternberg, and Lepidodendron elegans all came from the same horizon.

In Great Britain, Bergeria dilatata has been repeatedly recorded, under various names, throughout the Westphalian; i.e. Kidston's Lepidodendron landsburgii, which originated

Figure 11. Palynomorphs. a) In situ microspore of the Microspinosporites orbiculus type. Note laevigate proximal surface and a part of densely microspinate distal sculpture and elevated rays of the trilete mark. SEM. Scale bar $=10 \mu \mathrm{m}$. b) Lateral view on in situ megaspore of the Lagenoisporites rugosus type. Note laevigate sculpture and developed gula (left). SEM. Scale bar $=150 \mu \mathrm{m}$. c) Semi-lateral view of in situ microspore of the Microspinosporites orbiculus type (x 500). Note difference between microspinate sculpture of the distal surface (lower) and laevigate proximal surface (upper). d) Proximal view of in situ microspore of the Microspinosporites orbiculus type (x 500). e) Partial semi-lateral view of in situ microspore of the Microspinosporites orbiculus type (x 500). f) In situ megaspore of the Lagenoisporites rugosus type without gula. Scale bar $=260 \mu \mathrm{m}$. g) In situ megaspore of the Lagenoisporites rugosus type with gula. Scale bar $=260 \mu \mathrm{m}$. h) Detail of the surface of an in situ megaspore of the Lagenoisporites rugosus type. Note negative imprints of ubish bodies and small circular ubish bodies (upper edge). SEM. Scale bar $=22 \mu \mathrm{m}$. i) Semi-lateral view on in situ megaspores of the Lagenoisporites rugosus type with gula. Scale bar $=180 \mu \mathrm{m}$. j) Two in situ megaspores of the Lagenoisporites rugosus type. SEM. Scale bar $=200 \mu \mathrm{m}$. Repository: Institute of Geology, Academy of Sciences, Prague, Czech Republic. 


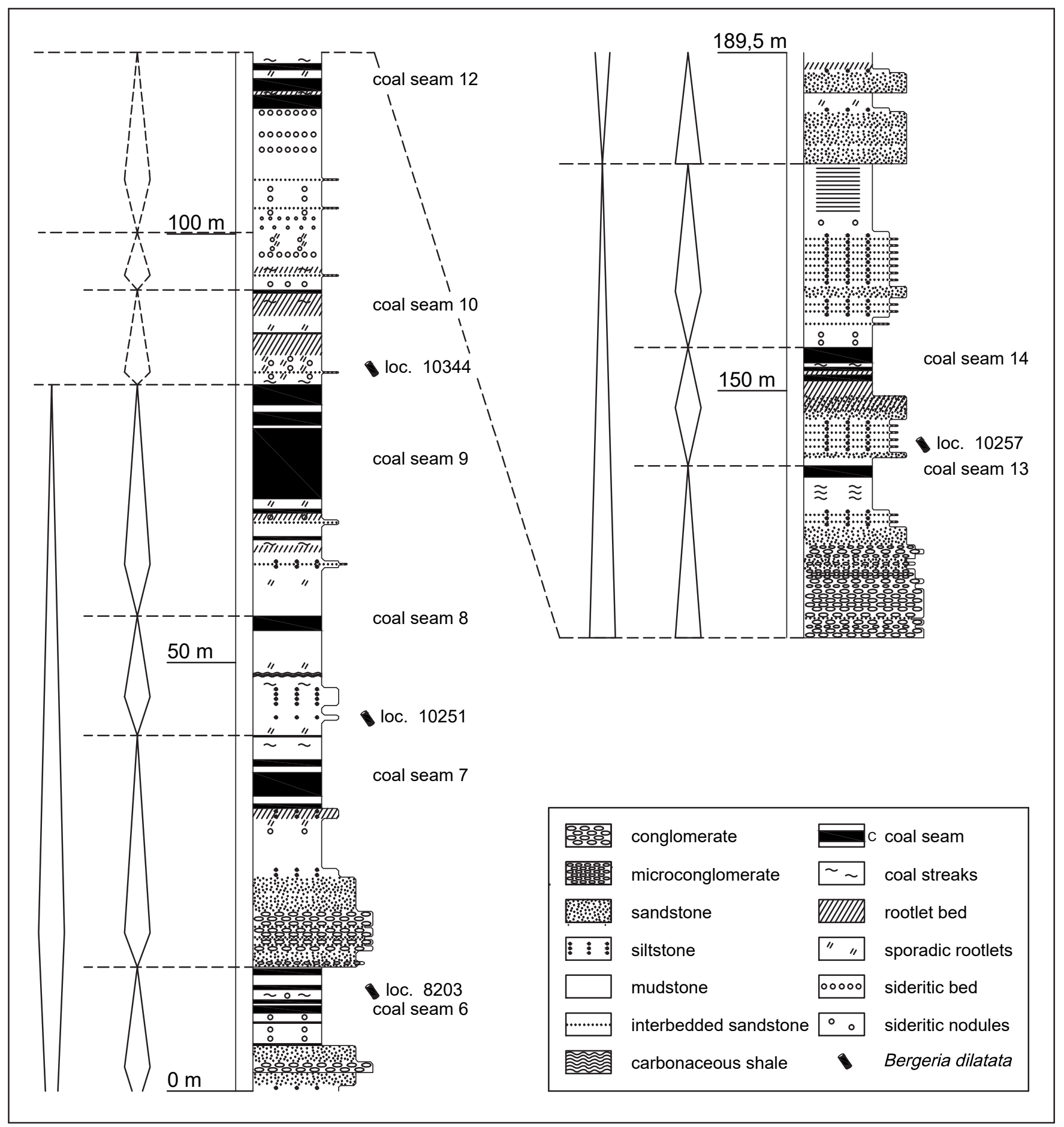

Figure 12. Stratigraphic column of part of the succession in Corta Ballesta showing the position of localities 8203, 10251, 10257 and 10344, from which most of the specimens figured here originated.

from the roof shales of the Whistler Seam, Kilmarnock, Ayrshire, Scotland, of lower Duckmantian age; the two specimens figured as Lepidodendron simile by Chaloner \& Boureau in Boureau (1967) also from lower Westphalian strata of Scotland (Clackmannanshire); the specimens figured as Lepidodendron ophiurus by Crookall (1964) from the Barnsley Coal, Yorkshire Coalfield, early Duckmantian, and from Hanley, North Staffordshire, upper Bolsovian; Cleal \& Thomas's (1988) Lepidodendron lycopodioides from the Cattybrook Claypit, on BristolSomerset Coalfield, upper Langsettian; or those figured by Crookall (1964) as Lepidodendron acutum, and as Lepidodendron acutum and Lepidodendron cf. lanceolatum by Bassett \& Edwards (1973, 1982), from the upper Bolsovian of Glamorgan, Wales. 
The westernmost occurrence of Bergeria dilatata is in the Michigan Basin, the United States of America, where Arnold (1949) figured it as Lepidodendron lanceolatum, Lepidodendron ophiuroides and Lepidodendron vestitum from upper Langsettian/upper Duckmantian strata. The species has also been recorded in the Warrior Basin, Alabama (Gillespie \& Rheams, 1985) and the Crab Orchand Mountains Formation, Georgia (Gillespie \& Crawford, 1985), from Langsettian strata. The records from West Virginia are those of Jongmans (1937) from Seam No. 3 of the Pocahontas Formation (lower Langsettian), Gillespie et al. (1978), who do not provide an exact locality, and Cross et al. (1996) from strata above the Eagle Coal of the Kanawha Formation, lower Duckmantian according to Blake et al. (2002). Lesquereux (1879-84) recorded the species at different levels in Illinois, Kentucky, Missouri and Pennsylvania. Lesquereux's types of Lepidodendron lanceolatum and Lepidodendron scutatum came from the Clinton Coal, Missouri, of early Asturian age. DiMichele et al.'s (2017) record as Synchysidendron originated in the roof shales of the Springfield Coal, Illinois Basin, of Asturian age.

Although Bell (1944) only figured one specimen of Bergeria dilatata (as Lepidodendron lanceolatum) from the Minas Basin, Nova Scotia, Canada, the review of the original collection by Álvarez-Vázquez \& Wagner (2014) indicates that it is a relatively frequent species in the upper Langsettian of the Cumberland Basin. Bell's (1938) records (under Lepidodendron lycopodioides) from the Sydney Basin, Nova Scotia, and those from the Minto Coalfield, New Brunswick (Bell, 1940, 1966 - as Lepidodendron ophiurus) came from (lower?) Bolsovian strata.

Records from the Nord/Pas-de-Calais Coalfield, northwestern France (Zeiller, 1886-88 - under Lepidodendron haidingeri, Lepidodendron lycopodioides, Lepidodendron ophiurus and Ulodendron majus) are from Duckmantian strata.

Lepidodendron gibbosum Sauveur (1848), and the specimens figured by him as Lepidodendron dilatatum and Lepidodendron sternbergii, originated from lower Westphalian strata of Belgium. Stockmans \& Willière's (1952) Ulodendron goodei came from Yeadonian strata. Van Amerom \& Lambrecht's (1979) Lepidodendron lycopodioides originated from lower Westphalian strata.

Records from South Limburg, the Netherlands, include material from Langsettian and Duckmantian strata (Jongmans, 1949, 1953; van der Veldt, 2008).

In the Ruhr Basin, western Germany, there are records of Bergeria dilatata (under different names) from Langsettian to Bolsovian (e.g. Gothan \& Remy, 1957; Drägert, 1964; Remy \& Remy, 1977).

Bergeria dilatata has been repeatedly recorded (under various names) from the Czech Republic. The two syntypes of Bergeria acuta (Presl in Sternberg, 1838; Kvaček \& Straková, 1997) originated from the Nýrany Member of the Kladno Formation, Central Bohemia, of Asturian age.
Presl's Bergeria angulata, Bergeria marginata, Bergeria quadrata and Bergeria rhombica also came from the same horizon. In the Radnice Basin there are records from the base of the Whetstone Horizon (Opluštil et al., 2009a) and from different levels of the Upper Radnice Coal (Ettingshausen, 1854; Pšenička \& Opluštil, 2011; Opluštil et al., 2016), of late Duckmantian age.

Romanian material from the Sirina Basin was initially regarded by Biţoianu (1972a, 1972b) as Westphalian D (= Asturian), but Wagner \& Álvarez-Vázquez (2008) indicate a Langsettian age.

In Bulgaria, Bergeria dilatata was recorded by Tenchov (1987) from Duckmantian and Bolsovian strata of the Svoge Basin.

Records published from South Briançonnais, French Alps, by Greber (1966) and Brousmiche-Delcambre et al. (1996, 1997) show Bergeria dilatata (under Lepidodendron lycopodioides and ?Tomiodendron) to range from Yeadonian to lower Langsettian.

Pšenička et al. (2012) ascribed the fossil plant collection (including Lepidodendron cf. acutum) from Val Sanagra, western Southern Alps, Italy, to the Duckmantian/ Bolsovian transition.

Bureau's (1913-14) Lycopodites foliosus and the other records from the Vendée, southwest France, refer to lower Westphalian strata.

According to Wagner \& Álvarez-Vázquez (2010), Bergeria dilatata ranges from Langsettian to lower Asturian in the Iberian Peninsula. In the Cantabrian Mountains of NW Spain, the sparse records are from uppermost Langsettian strata of La Camocha, Asturias (Wagner \& Álvarez-Vázquez, 1995) and San Emiliano, León (Wagner \& Bowman, 1983), and from lower Asturian strata of the Central Asturian Coalfield. In SW Spain, the species has been widely sampled in upper Langsettian/ lower Duckmantian and lower Bolsovian strata of the Peñarroya-Belmez-Espiel Coalfield (Álvarez-Vázquez, 1995; Wagner \& Álvarez-Vázquez, 2010; this paper).

Bergeria dilatata has also been published under different names from Bolsovian strata in Djerada, Morocco (Jongmans, 1952) and the Sud-Oranais, Algeria (Jongmans, 1951; Migier, 1982, 1997).

Bergeria dilatata is, therefore, a common, widespread and long-ranging species that has been recorded (under various names) from Yeadonian to Asturian strata. It has been reported most often from the Langsettian, Duckmantian and Bolsovian substages.

\section{GENERAL CONCLUSIONS}

This work recognizes the widespread presence of the arborescent lycopsid Bergeria dilatata in the Westphalian successions of North America, Europe and North Africa. 
Considerable confusion has surrounded identification of this species. The very abundant material from the Peñarroya-Belmez-Espiel Coalfield has allowed for a welldocumented record of the variability of the species and the recognition of extensive synonymous records.

The priority of the genus Bergeria for lycopsid stem adpressions characterized by false leaf scars was noted by Álvarez-Vázquez \& Wagner (2014). The present work documents the range of the Spanish material from the Peñarroya-Belmez-Espiel Coalfield and provides for the first time a photograph of the type of Lindley \& Hutton's Lepidodendron dilatatum, housed in the Hutton Collection, Newcastle-upon-Tyne. In conjuction, it has also been possible to recognize further illustrations attributable to the genus Bergeria and to designate two new combinations: Bergeria lycopodioides and Bergeria wingfieldense.

Palynological study of strobili closely associated with Bergeria dilatata has confirmed their identification as Flemingites russelianus, a bisporangiate cone taxon; this has also been previously recognized in the literature (Chaloner, 1953) as corresponding to adpression material identifiable as Bergeria dilatata. The present work strongly supports the whole-plant relationship between Bergeria dilatata and Flemingites russelianus.

\section{ACKNOWLEDGEMENTS}

The authors are particularly pleased to present this work in a volume dedicated to recognise the life work of Dr M.L. Martínez Chacón (Lis) and Dr L.C. Sánchez de Posada (Suco); for those of the authors working in Spain, they have consistently been inspiring and supportive friends and colleagues, and all the authors recognise the importance of their open welcome and helpfulness to fellow workers. The curatorial staff, and in particular Ms. Sylvia Humphrey, of the Great North Museum-Hancock, Newcastle-upon-Tyne, England, are thanked for enthusiastic assistance in making the Hutton collection available for inspection. Juan I. Peláez and mining engineers at ENCASUR Company have been most helpful in providing access to boreholes and collecting sites. Carmen Álvarez-Vázquez wishes to acknowledge unconditional field support from Juan Peláez and Eva Peláez Álvarez. The authors acknowledge with thanks the helpful and well-focused corrections and suggestions of the two referees, Bill DiMichele and Arden Bashforth. Jiří Bek acknowledges financial support from the Research Program of the Institute of Geology AS CR, v.v.i. (RVO67985831).

\section{REFERENCES}

Álvarez-Vázquez, C. 1995. Macroflora del Westfaliense inferior de la cuenca de Peñarroya-Belmez-Espiel (Córdoba). Doctoral Thesis, Department of Geology, University of Oviedo (unpublished).

Álvarez-Vázquez, C. 1999. Nuevos datos aportados por la macroflora a la datación y conocimiento de la cuenca carbonífera de Peñarroya-Belmez-Espiel (Córdoba, SO España). Trabajos de Geología, 21 (Volumen homenaje a J. Truyols), 37-46.

Álvarez-Vázquez, C. \& Cleal, C.J. 2016. Wagneropteris minima, a new medullosalean pteridosperm from the middle Westphalian (Middle Pennsylvanian) of the Peñarroya-Belmez-Espiel Coalfield (Córdoba province, SW Spain). Spanish Journal of Palaeontology, 31, 5-24.

Álvarez-Vázquez, C. \& Wagner, R.H. 2014. Lycopsida from the lower Westphalian (Middle Pennsylvanian) of the Maritime Provinces, Canada. Atlantic Geology, 50, 167232; doi: 10.4138/atlgeol.2014.011.

Álvarez-Vázquez, C., Bek, J., Drábková, J. \& Wagner, R.H. in prep. A new record of heterosporous Polysporia Newberry from the middle Westphalian of the Peñarroya Basin, SW Spain, its spore contents and a discussion of Palaeozoic and Mesozoic isoetaleans.

Amerom, H.W.J. van 1981. Over mijnsteenstorten, steenkoolflora en -fauna. Miscellanea Geologica Coriovallana, 73-80.

Amerom, H.W.J. van \& Kabon, H. 2003. Neue fossile Floren aus dem Nötscher Karbon (3. Teil). Carinthia II, 193/113, 527-560.

Amerom, H.W.J. van \& Lambrecht, L. 1979. Geologische und Paläobotanisch-Palökologische Untersuchungen im Westfal A und B von Lüttich (Belgien). Huitième Congrès International de Stratigraphie et Géologie du Carbonifère, Moscow 1975, 3, 148-158.

Andrews, H.N. 1955. Index of Generic Names of Fossil Plants, 1820-1965. Geological Survey Bulletin 1300, $1-354$.

Anisimova, O.I. 1979. Flora and Phytostratigraphy of the Middle Carboniferous of North Caucasus. Akademia Nauk, Ukrainskoy SSR. Institut Geologicheskikh, Naukova Dumka, Kiev, 1-108 (in Russian).

Arber, E.A.N. 1903a. The Fossil Flora of the Cumberland Coalfield, and the Palaeobotanical Evidence with regard to the Age of the Beds. Quarterly Journal of the Geological Society, London, LIX, 1-22.

Arber, E.A.N. 1903b. Notes on Fossil Plants from the Ardwick Series of Manchester. Memoirs and Proceedings of the Manchester Literary and Philosophical Society, XLVIII, 1-33.

Arber, E.A.N. 1912. On the Fossil Flora of the Forest of Dean Coalfield (Gloucestershire), and the Relationships of the Coalfields of the West of England and South Wales. Philosophical Transactions of the Royal Society of London, Series B, CCII, 233-281.

Arber, E.A.N. 1914. On the Fossil Floras of the Wyre Forest, with Special Reference to the Geology of the Coalfield 
and its Relationships to the Neighbouring Coal Measure Areas. Philosophical Transactions of the Royal Society of London, Series B, CCIV, 363-445.

Arber, E.A.N. 1922. Critical Studies of Coal-measure Plant impressions. Journal of the Linnean Society, Botany, XLVI, 171-217; doi: 10.1111/j.1095-8339.1922.tb00484.x.

Arnold, C.A. 1949. Fossil flora of the Michigan Coal Basin. Contributions from the Museum of Paleontology, University of Michigan, VII, 131-269.

Balme, B.A. 1995. Fossil in situ spores and pollen grains: an annotated catalogue. Review of Palaeobotany and Palynology, 87, 81-323; doi: 10.1016/00346667(95)93235-X.

Bassett, M.G. \& Edwards, D. 1973. Fossil Plants from Wales. Amgueddfa, Bulletin of the National Museum of Wales, $13,1-27$.

Basset, M.G. \& Edwards, D. 1982. Fossil Plants from Wales. Amgueddfa, Bulletin of the National Museum of Wales, Geological Series, 2, 1-42.

Bateman, R.M. \& DiMichele, W.A. 1991. Hizemodendron, gen. nov., a Pseudoherbaceous Segregate of Lepidodendron (Pennsylvanian): Phylogenetic Context for Evolutionary Changes in Lycopsid Growth Architecture. Systematic Botany, 16, 195-205.

Bek, J. 2013. Microspinosporites, a new genus of Palaeozoic pseudosaccate miospores of flemingitalean affinity. Bulletin of Geosciences, 88, 573-581; doi: 10.3140/bull. geosci.1365.

Bek, J. 2017. Paleozoic spores and pollen. Lycopsida. Palaeontographica, Abt. B, 296, 1-111; doi: 10.1127/ palb/2017/0053.

Bell, W.A. 1938. Fossil Flora of Sydney Coalfield, Nova Scotia. Canada Department of Mines and Resources, Mines and Geology Branch, Geological Survey, Memoir 215, 1-334.

Bell, W.A. 1940. The Pictou Coalfield, Nova Scotia. Canada Department of Mines and Resources, Mines and Geology Branch, Geological Survey, Memoir 225, 1-161.

Bell, W.A. 1944. Carboniferous rocks and fossil floras of northern Nova Scotia. Canada Department of Mines and Resources, Mines and Geology Branch, Geological Survey, Memoir 238, 1-119.

Bell, W.A. 1962. Flora of Pennsylvanian Pictou Group of New Brunswick. Geological Survey of Canada, Department of Mines and Technical Surveys, Bulletin 87, 1-71.

Bell, W.A. 1966. Illustrations of Canadian Fossils Carboniferous Plants of Eastern Canada. Geological Survey of Canada, Department of Mines and Technical Surveys, Paper 66-11, pls I-XXXVI.

Binney, E.W. 1871. Observations on the structure of fossil plants found in the Carboniferous strata. pt. II. Lepidostrobus and some allied cones. Palaeontographical Society Monograph, 33-62.

Biţoianu, C. 1972a. Observaţii asupra conţinutului paleobotanic al depozitelor carbonifere din zona Baia Nouă (Banat). Studii Cercetări de Geologie, Geofizică, Geografie, Seria Geologie, 17, 125-130.
Biţoianu, C. 1972b. Conţinutul floristic al depozitelor carbonifere din zona Sviniţa. Studii Cercetări de Geologie, Geofizică, Geografie, Seria Geologie, 17, 391-399.

Biţoianu, C. 1973. La flore du Carbonifère supérieur de la Roumanie. Septième Congrès International de Stratigraphie et Géologie du Carbonifère, Krefeld 1971, II, 115-127.

Blake, B.M., Cross, A.T., Eble, C.F., Gillespie, W.H. \& Pfefferkorn, H.W. 2002. Selected plant megafossils from the Carboniferous of the Appalachian region, eastern United States: geographic and stratigraphic distribution. In: Carboniferous and Permian of the World: Proceedings XIV International Congress Carboniferous and Permian Stratigraphy, Calgary, Alberta 1999. (eds. Hills, L.V, Henderson, C.M. \& Bamber, E.W.). Canadian Society of Petroleum Geologists, Memoir 19, 259-335.

Boulay, N. 1876. Le terrain houiller du Nord de la France et ses végétaux fossiles. Thèse de Géologie, Faculté des Sciences de Caen, Imprimérie Lefebvre-Ducrocq, Lille, $74 \mathrm{p}$.

Boureau, E. 1967. Traité de Paléobotanique. T. II. JovetAst, S. - Prémier Embranchement. Bryophyta: 19-190; Höeg, O.A. - Deuxième Embranchement. Psilophyta: 191-433; Chaloner, W.G. \& Boureau, E. - Troisième Embranchement. Lycophyta: 435-802. Masson et Cie, Paris.

Brack-Hanes, S.D. \& Thomas, B.A. 1983. A re-examination of Lepidostrobus Brongniart. Botanical Journal of the Linnean Society, 86, 125-133; doi: 10.1111/j.10958339.1983.tb00720.x.

Brongniart, A. 1822. Sur la classification et la distribution des végétaux fossiles en général, et sur ceux des terrains de sédiment supérieur en particulier. Mémoires du Musée d'Histoire naturelle Paris, 8, 203-240.

Brongniart, A. 1828-1838. Histoire des Végétaux Fossiles, ou Recherches Botaniques et Géologiques sur les Végétaux Renfermés dans les Diverses Couches du Globe. Texte: I (1828-1837) - 1 (1828): i-xii, 1-80; 2 (1828): 81-136; 3 (1829): 137-168; 4 (1830): 169-208; 5 (1831): 209-248; 6 (1831): 249-264; 7 (1833): 265-288; 8 (1834): 289-312; 9 (1834): 313-316; 10 (1836): 337-368; 11 (1837): 369-416; 12 (1837): 417-488. II (1837-1838) - 13 (1837): 1-24; 14 (1838): 25-56; 15 (1838): 57-72; Atlas: I - pls 1-166; II - pls 1-30. Facsimile Edition W. Junk, 18, Berlin (1915).

Brousmiche Delcambre, Cl., Coquel, R. \& Mercier, D. 1997. Sur l'âge des terrains carbonifères affleurant au col de Tramouillon (Massif de Gaulent, Sud Briançonnais). Revue Paléobiologie, Genève, 16, 169-179.

Brousmiche Delcambre, Cl., Mercier, D. \& Coquel, R. 1996. Révision de la flore carbonifère du Briançonnais. Implications stratigraphiques. Ière partie: Vallée de la Durance au sud de Briançon. Palaeontographica, Abt. $B, 239,77-107$.

Brousmiche Delcambre, Cl., Mercier, D. \& Coquel, R. 1998. Révision de la flore carbonifère du Briançonnais. Implications stratigraphiques. Partie II: Le Nord de Briançon, de la vallée de la Guisane à celle de la Clarée. Palaeontographica, Abt. B, 248, 87-125. 
Bureau, M.E. 1913-1914. Bassin de la Basse Loire. Fascicule II: Decription des flores fossiles. Études des Gîtes minéraux de la France. Texte (1914): 1-417. Atlas (1913): pls I-LXXX.

Carruthers, W. 1865. On an undescribed cone from the Carboniferous beds of Airdrie, Lanarkshire. Geological Magazine, 2, 433-440.

Chaloner, W.G. 1953. On the Megaspores of Four Species of Lepidostrobus. Annals of Botany, N.S., XVII (66), 263293; doi: 10.1093/oxfordjournals.aob.a083350.

Chaloner, W.G. \& Meyer-Berthaud, B. 1983. Leaf and stem growth in the Lepidodendrales. Botanical Journal of the Linnean Society, 86, 135-148; doi: 10.1111/j.10958339.1983.tb00721.x.

Chaloner, W.G., Leistikow, K.U. \& Hill, A. 1979. Brasilodendron gen. nov. and B. pedroanum (Carruthers) comb. nov., a Permian lycopod from Brazil. Review of Palaeobotany and Palynology, 28, 117-136; doi: 10.1016/0034-6667(79)90004-6.

Cleal, C.J. \& Thomas, B.A. 1988. The Westphalian fossil floras from the Cattybrook Claypit, Avon (Great Britain). Geobios, 21, 409-433; doi: 10.1016/S00166995(88)80043-3.

Cleal, C.J. \& Thomas, B.A. 1994. Plant Fossils of the British Coal Measures. Palaeontological Association, Field Guides to Fossils, 6, 1-222.

Cleal, C.J., Lazarus, M. \& Townsend, A. 2005. Illustrations during the "Golden Age" of palaeobotany: 1800-1840. In: History of Palaeobotany: Selected Essays (eds. Bowden, A.J., Burek, C.V. \& Wilding, R.). Geological Society, London, Special Publications, 241, 41-61; doi: 10.1144/ GSL.SP.2003.207.01.05.

Crookall, R. 1925. On the Fossil Flora of the Bristol and Somerset Coalfield. Part II. Geological Magazine, LXII, 385-410.

Crookall, R. 1929. Coal Measure Plants. Edward Arnold \& Co., London, 80 p.

Crookall, R. 1932. The Relative Value of Fossil Plants in the Stratigraphy of the Coal Measures. Memoirs \& Proceedings of the Manchester Literary and Philosophical Society, LXXVI (1931-32), 91-122.

Crookall, R. 1964. Fossil Plants of the Carboniferous Rocks of Great Britain. (Second Section). Memoirs of the Geolological Survey of Great Britain, Palaeontology, IV. Part 3: vii-xii, 217-354, pls LIX-LXXXI.

Crookall, R. 1966. Fossil Plants of the Carboniferous Rocks of Great Britain. (Second Section). Memoirs of the Geolological Survey of Great Britain, Palaeontology, IV. Part 4: xiii-Xx, 355-571, pls LXXXII-CVI.

Cross, A.T., Gillespie, W.H., \& Taggart, R.E. 1996. Upper Paleozoic vascular plants. In: Fossils of Ohio (eds. Feldmann, R.M. \& Hackathorn, M.). Department of Natural Resources, Division of Geological Survey, Bulletin 70, 396-479.

Daber, R. 1969. Paläobotanische Hinweise auf eine paralisch beeinflußte Oberkarbon-Senke im tieferen Untergrund Nordostdeutschlands, II (Teil 1). Geologie, 18, 253-297.
Dijkstra, S.J. \& Amerom, H.W.J. van 1991. Fossilium Catalogus. II: Plantae. Pars 94: Lycopodiales X. 2. Supplement. Kugler Publications, Amsterdam, 1-127.

Dijkstra, S.J. \& Amerom, H.W.J. van 1994. Fossilium Catalogus. II: Plantae. Pars 95: Lycopodiales XI. 2. Supplement und Nachtrag zum 2. Supplement. Kugler Publications, Amsterdam, 129-296.

DiMichele, W.A. 1980. Paralycopodites Morey \& Morey, from the Carboniferous of Euramerica - a reassessment of generic affinities and evolution of "Lepidodendron" brevifolium Williamson. American Journal of Botany, 67, 1466-1476.

DiMichele, W.A. 1985. Diaphorodendron, gen. nov., a Segregate from Lepidodendron (Pennsylvanian Age). Systematic Botany, 10, 453-458; doi: 10.2307/2419138.

DiMichele, W.A. \& Bateman, R.M. 1992. Diaphorodendraceae, fam. nov. (Lycopsida: Carboniferous): Systematics and evolutionary relationships of Diaphorodendron and Synchysidendron, gen. nov. American Journal of Botany, 79, 605-617.

DiMichele, W.A. \& Bateman, R.M. 1993. Validation of Synchysidendron, gen. nov. (Fossiles). Taxon, 42, 647-648.

DiMichele, W.A. \& Phillips, T.L. 1985. Arborescent lycopod reproduction and palaeoecology in a coal-swamp environment of Late Middle Pennsylvanian age (Herrin Coal, Illinois, U.S.A.). Review of Palaeobotany and Palynology, 44, 1-26; doi: 10.1016/0034-6667(85)90026-0.

DiMichele, W.A. \& Phillips, T.L. 1994. Paleobotanical and paleoecological constraints on models of peat formation in the Late Carboniferous of Euramerica. Palaeogeography, Palaeoclimatology, Palaeoecology, 106, 39-90; doi: 10.1016/0031-0182(94)90004-3.

DiMichele, W.A., Elrick, S.D. \& Nelson, W.J. 2017. Vegetational zonation in a swamp forest, Middle Pennsylvanian, Illinois Basin, U.S.A., indicates niche differentiation in a wetland plant community. Palaeogeography, Palaeoclimatology, Palaeoecology, 487, 71-92; doi: 10.1016/j.palaeo.2017.08.020.

Drägert, K. 1964. Pflanzensoziologische Untersuchungen in den Mittleren Essener Schichten des nördlichen Ruhrgebietes. Forschungsberichte des Landes NordrheinWestfalen, 1363, 1-295.

Dybová-Jachowicz, S., Jachowicz, A., Karczewska, J., Lachkar, G., Loboziak, S., Piérart, P., Turnau, E. \& Zoldani, Z. 1979. Note préliminaire sur la révision des mégaspores à gula du Carbonifère. Les príncipes de la classification. Acta Palaeontologica Polonica, 24, 411-422.

Dybová-Jachowicz, S., Jachowicz, A., Karczewska, J., Lachkar, G., Loboziak, S., Piérart, P., Turnau, E. \& Zoldani, Z. 1982. Révision des mégaspores à gula du Carbonifére, Part 1. Prace Instytutu Geologicznego, CVII, 5-44.

Dybová-Jachowicz, S., Jachowicz, A., Karczewska, J., Lachkar, G., Loboziak, P., Piérart, S., Turnau, E. \& Zoldani, Z. 1987a. Revision of Carboniferous megaspores with gula, Part II. Prace Instytutu Geologiznego, CXV, 5-31.

Dybová-Jachowicz, S., Jachowicz, A., Karczewska, J., Lachkar, G., Piérart, P., Loboziak, S., Turnau, E. \& Zoldani, Z. 
1987b. Revision of Carboniferous megaspores with gula, Part III. Prace Instytutu Geologicznego, CXXI, 5-49.

Eichwald, E. 1859-60. Lethaea Rossica ou Paléontologie de la Russie. Prémier Volumen. Première Section de l'Ancienne Période, Contenant la Flore de l'Ancienne Période et la Faune jusqu'aux Mollusques. Atlas (1959): pls I-LIX. Text (1860): 681 p. Librairie de E. Schweizerbart, Stuttgart.

Ettingshausen, C. von 1854. Die Steinkohlenflora von Radnitz in Böhmen. Abhandlugen der kaiserlichen-königlichen geologischen Reichsanstalt, II, 3, 1-74.

Feistmantel, O. 1875. Die Versteinerungen der böhmischen Kohlenablagerungen. In: Beiträge zur Naturgeschichte der Vorwelt (eds. W. Dubker \& K.A. Zittel). Palaeontographica, XXIII (4-6), 173-222.

Fischer, F.F. 1905. Lepidodendron - Erhaltungszustand. Bergeria Presl zum Teil. In: Abbildungen und Beschreibungen fossiler Pflanzen-Reste (ed. Potonié, H.). Königliche Preußische Geologische Landesanstalt, Lieferung III, 42, 1-12.

Gillespie, W.H. \& Crawford, T.J. 1985. Plant megafossils from the Carboniferous of Georgia, U.S.A. Dixième Congrès International de Stratigraphie et Géologie du Carbonifère, Madrid 1983, 2, 247-256.

Gillespie, W.H. \& Rheams, L.J. 1985. Plant megafossils from the Carboniferous of Alabama, U.S.A. Dixième Congrès International de Stratigraphie et Géologie du Carbonifère, Madrid 1983, 2, 191-202.

Gillespie, W.H., Clendening, J.A. \& Pfefferkorn, H.W. 1978. Plant fossils of West Virginia and adjacent areas. West Virginia Geological and Economic Survey, Educational Series ED-3A, 1-172.

Göppert, H.R. 1836. Die fossilen Farrnkräuter. Verhandlungen der Kaiserlichen Leopoldinisch-Carolinischen Akademie der Naturforscher, XVII Supplement, 486 p.

Gothan, W. \& Franke, F. 1929. Der Westfälisch-Rheinische Steinkohlenwald und seine Kohlen. Wissenschaftliche Heimatbücher für den Westfälisch-Rheinischen Industriebezirk, Band II, 1-141.

Gothan, W. \& Remy, W. 1957. Steinkohlenpflanzen. Leitfaden zum Bestimmen der Wichtigsten Pflanzlichen Fossilien des Paläozoikums im Rheinisch-Westfälischen Steinkohlengebiet. Verlag Glückauf GMBH, Essen, 248 p.

Greber, Ch. 1966. Flore et stratigraphie du Carbonifère des Alpes françaises. Mémoires du Bureau de Recherches Géologiques et Minières, Série A, 21, 1-380.

Hörich, O. 1916. Phialophloios quadratus, eine neue Lepidophytengattung. Jahrbuch Königlichen Preussisschen Geologische Landesanstalt, XXXV (1914), II, 426-430.

Howse, R. 1888. A Catalogue of Fossil Plants from the Hutton Collection, Presented by the Council of the Mining Institute to the Natural History Society, 1883. Natural History Transactions of Northumberland, Durham, and Newcastle-upon-Tyne, X, 1-135.

Janssen, R.E. 1940. Some fossil plant types of Illinois. A Restudy of the Lesquereux types in the Worthen Collection of the Illinois State Museum, augmented by descriptions of new species from Mazon Creek. Illinois State Museum, Scientific Papers, I, 1-124.
Janssen, R.E. 1957. Leaves and stems from fossil forests. A handbook of the paleobotanical collections in the Illinois State Museum. Illinois State Museum, Popular Science Series, I, 1-190. (First printing, 1939).

Jongmans, W.J. 1909. The flora of the Dutch Carboniferous compared with that of the adjacent coalfields. In: Waterschoot van der Gracht, A.J.M van. The deeper geology of the Netherlands and adjacent regions, with special reference to the latest borings in the Netherlands, Belgium and Westphalia. Memoirs of the Government Institute for the Geological Explorations of the Netherlands (Rijksopsporing van Delfstoffen), 2, 1-437.

Jongmans, W.J. 1913. Fossilium Catalogus. II: Plantae. Pars 1: Lycopodiales I. W. Junk, Berlin: 1-52.

Jongmans, W.J. 1929. Fossilium Catalogus. II: Plantae. Pars 15: Lycopodiales II. W. Junk, Berlin: 55-525.

Jongmans, W.J. 1936. Fossilium Catalogus. II: Plantae. Pars 21: Lycopodiales V (incl. Hydropteridae, Psilophytales, Sphenophyllales). W. Junk, Berlin: 999-1188.

Jongmans, W.J. 1937. Comparison of the floral succession in the Carboniferous of West Virginia with Europe. Deuxième Congrès pour l'avancement des études de Stratigraphie Carbonifère, Heerlen 1935, I, 393-415.

Jongmans, W.J. 1949. Het wisselend aspect van het bos in de oudere geologische formaties. In: Hout in alle tijden (ed. Boerhave Beekman, W.). Deel I, Hoofdstuk 1, 1-164.

Jongmans, W.J. 1951. La flore du Westphalien supérieur. In: Les bassins houillers du Sud-Oranais. Livre II. Contribution à l'étude paléontologique (Jongmans, W.J. \& Deleau, P.C.). Bulletin du Service de la carte géologique de 1'Algérie, $1^{\text {re }}$ série, Paléontologie, 13, 1-25 (flore), 27-48 (faune).

Jongmans, W.J. 1952. Note sur la flore du terrain carbonifère de Djerada (Maroc oriental). Notes et Mémoires, Service géologique, Division des Mines et de la Géologie, Direction de la production industrielle et de Mines, Maroc, 91, 1-29.

Jongmans, W.J. 1953. Palaeontology of the sections in the Lower Westphalian A, in borings LXVI, LXVII, LXVIII in Limburg (Voerendaal). Flora. Mededelingen van de Geologische Stichting, Serie C-III-1 (3), 29-45.

Jongmans, W.J. \& Dijkstra, S.J. 1969. Fossilium Catalogus. II: Plantae. Pars 72-73: Lycopodiales VII. Supplement. Ed. W. Junk, Netherlands, 1333-1547.

Jonker, F.P. 1976. The Carboniferous "genera" Ulodendron and Halonia - An assessment. Palaeontographica, Abt. $B, 157$ (5-6), 97-111.

Josten, K.H. 1983. Die fossilen Floren im Namur des Ruhrkarbons. Fortschritte in der Geologie von Rheinland und Westfalen, 31. Text-Band: 1-327, 112 Abb. TafelBand.: Tafn. 1-56.

Kidston, R. 1885. On the relationship of Ulodendron, Lindley \& Hutton, to Lepidodendron, Sternberg; Bothrodendron, Lindley \& Hutton; Sigillaria, Brongniart; and Rhytidodendron, Boulay. Annals and Magazine of Natural History, XVI, 123-179, 239-260.

Kidston, R. 1887. On the fossil flora of the Radstock Series of the Somerset and Bristol Coal Field (Upper Coal 
Measures). Part I. Transactions of the Royal Society of Edinburgh, XXXIII, Part II, 335-417.

Kidston, R. 1891. Notes on the Palaeozoic species mentioned in Lindley and Hutton's "Fossil Flora". Proceedings of the Royal Physical Society, Edinburgh, X (1889-90), 345-391.

Kidston, R. 1893. On the fossil plants of the Kilmarnock, Galston, and Kilwinning Coal Fields, Ayrshire. Transactions of the Royal Society of Edinburgh, XXXVII, Part II (16), 307-358.

Kidston, R. 1903. The fossil plants of the Carboniferous rocks of Canonbie, Dumfriesshire, and of parts of Cumberland and Northumberland. Transactions of the Royal Society of Edinburgh, XL, Part IV (31), 741-833.

Kidston, R. 1911. Les Végétaux Houillers Recueillis dans le Hainaut Belge et se Trouvant dans les Collections du Musée Royal d'Histoire Naturelle à Bruxelles. Imprimerie Polleunis \& Ceuterick, Bruxelles, 282 p.

Kvaček, Z. \& Kvaček, J. 1992. Šternberkovo dílo Flora der Vorwelt, jeho význam a stav dokladové sbírky. [Sternberg's work Flora der Vorwelt, its significance and the state of the original collection]. Časopis Národního Muzea v Praze, 158, 31-42.

Kvaček, J \& Straková, M. 1997. Catalogue of Fossil Plants Described in Works of Kaspar M. Sternberg. National Museum, Prague, $201 \mathrm{p}$.

Laveine, J.-P. 1989. Guide Paléobotanique dans le Terrain Houiller Sarro-Lorrain. Texte: 1-154. Atlas: pls 1-64. Documents des Houillères du Bassin de Lorraine. Imprimerie des Houillères du Bassin de Lorraine.

Laveine, J.-P., Zhang, S., Liu, L., Gu, F., Liu, Y., Wen, J. \& Lemoigne, Y. 2003. The Late Carboniferous floras of the Delingha area (Wulan County, Qinghai Province, Northwest China). Revue Paléobiologie, Genève, 22, 567-651.

Lesquereux, L. 1866. An enumeration of the fossil plants found in the Coal Measures of Illinois, with descriptions of the new species. Geological Survey of Illinois, Paleontology, II, 427-470.

Lesquereux, L. 1879-1884. Description of the Coal Flora of the Carboniferous Formation in Pennsylvania and throughout the United States. Second Geological Survey of Pennsylvania, Report of Progress. Text - I (1880): 1-354; II (1880): 355-694, i-lxiii, pls LXXXVI, LXXXVII; III (1884): 695-977, pls LXXXVIII-CXI. Atlas (1879): pls A, B; pls I-LXXXV.

Lindley, J. \& Hutton, W. 1831-1837. The Fossil Flora of Great Britain; or, Figures and Descriptions of the Vegetable Remains found in a Fossil State in this Country. I (1831-1833) - 1831: 1-47, pls 1-14; 1832: 48-166, pls 1559; 1833: 167-224, pls 60-79; II (1833-1835) - 1833: 1-56, pls 80-99; 1834: 57-156, pls 100-137; 1835: 157-208, pls 138-156; III (1835-1837) - 1835: 1-72, pls 157-176; 1836: 73-122, pls 177-194; 1837: 123-208, pls 195-230. James Ridgway and Sons, London.

Mammatt, E. 1834. A Collection of Geological Facts and Practical Observations, Intended to Elucidate the Formation of the Ashby Coal-Field, in the Parish of Ashby-de-la-Zouch and the Neighbouring District; Being the Result of Forty Years Experience and Research. G. Lawford Publisher, London, $101 \mathrm{p}$.

Maithy, P.K. 1972. Fossil flora of Westphalian D from Piesberg near Osnabrück. Palaeontographica, Abt. B, 139, 83-104.

Meyen, S.V. 1972. Are there ligula and parichnos in Angara Carboniferous lepidophytes? Review of Palaeobotany and Palynology, 14, 149-157; doi: 10.1016/00346667(72)90016-4.

Meyen, S.V. 1976. Carboniferous and Permian lepidophytes of Angaraland. Palaeontographica, Abt. B, 157, 112-157.

Migier, T. 1972. Charakterystyka florystyczna karbonu produktywnego Górnośląskiego Zagłębia Węglowego. [A floristic description of the Productive Carboniferous of the Upper Silesian Coal Basin]. Instytut Geologiczny, Prace, LXI, 135-182. (in Polish with English summary).

Migier, T. 1982. Profil utworów westfalu C-D basenu Mezarif w Algierii. [The Westphalian C-D sequence of the Mezarif Basin in Algeria]. Biuletyn Instytutu Geologicznego, 338, 23-70 (in Polish with English summary).

Migier, T. 1997. Carboniferous flora in South Oran Basins in Algeria. In: Proceedings of the XIII International Congress on the Carboniferous and Permian, Kraków, Poland, 1995. Prace Państwowego Instytutu Geologicznego, CLVII, Part 1, 259-268.

Morey, E.D. \& Morey, P.R. 1977. Paralycopodites minutissimum gen. et sp. n. from the Carbondale Formation of Illinois. Palaeontographica, Abt. B, 162, 64-69.

Němejc, F. 1942. Paläobotanische Studien im Gebiete des westbulgarischen Permokarbons. Geologického Ústavu Pro Čechy a Moravu, XVIII (3), 125-156.

Němejc, F. 1947. The Lepidodendraceae of the coaldistricts of Central Bohemia (A preliminary study). Sbornik Národniho Musea v Praze (Acta Musei nationalis Pragae), III.B (2), Geologia et Palaeontologia, 2, 45-87.

Novik, E.O. 1952. Coal measure flora of the European part of SSSR. Akademia Nauk SSSR, I, 1-468 (in Russian).

Novik, E.O. 1954. Carboniferous flora of the eastern part of the Donetz Basin. Akademia Nauk, Ukrainskoi SSR. Trudi Instituta Geologicheskikh Nauk, Serie Stratigrafii $i$ Paleontologii, vipusk, 7, 1-128 (in Russian).

Opluštil, S. \& Bek, J. 2009. Some Pennsylvanian arborescent lycopsid cones and their microspores from the British coalfields. Bulletin of Geosciences, 84, 203-226.

Opluštil, S., Pšenička, J., Libertín, M. \& Šimůnek, Z. 2007. Vegetation patterns of Westphalian and Lower Stephanian mire assemblages preserved in tuff beds of the continental basins of Czech Republic. Review of Palaeobotany and Palynology, 143, 107-154; doi: 10.1016/j.revpalbo.2006.06.004.

Opluštil, S., Pšenička, J., Šimůnek, Z. \& Libertín, M. 2016. Floras of clastic and peat-forming Pennsylvanian wetlands: are they different? A case study from the Upper Radnice Coal (late Duckmantian), Kladno Coalfield, Czech Republic. Spanish Journal of Palaeontology, 31, 145-180.

Opluštil, S., Šimůnek, Z., Pšenička, J., Bek, J. \& Libertín, M. 2017. A 25 million year of macrofloral record (Carboniferous-Permian) in the Czech part of the 
Intra-Sudetic Basin; biostratigraphy, plant diversity and vegetation patterns. Review of Palaeobotany and Palynology, 244, 241-307; doi: 10.1016/j. revpalbo.2016.11.011.

Opluštil, S., Pšenička, J., Libertín, M., Bashforth, A.R., Šimůnek, Z., Drábková, J. \& Dăsková, J. 2009a. A Middle Pennsylvanian (Bolsovian) peat-forming forest preserved in situ in volcanic ash of the Whetstone Horizon in the Radnice Basin, Czech Republic. Review of Palaeobotany and Palynology, 155, 234-274; doi: 10.1016/j.revpalbo.2009.03.002.

Opluštil, S., Pšenička, J., Libertín, M., Bek, J., Dǎsková, J., Šimůnek, Z. \& Drábková, J. 2009b. Composition and structure of an in situ Middle Pennsylvanian peat-forming plant assemblage buried in volcanic ash, Radnice Basin (Czech Republic). Palaios, 24, 726-746; doi: 10.2110/ palo.2008.p08-128r.

Owen, D.D. 1852. Report of a Geological Survey of Wisconsin, Iowa, and Minnesota; and Incidentally of a Portion of Nebraska Territory. Made under instructions from the United States Treasury Department, Lippincolt, Grambo \& Co., Philadelphia, 638 p.

Pearson, H.L. 1986. Structure and taxonomy of the Carboniferous lycopsid Anabathra. Bulletin British Museum natural History (Geology), 40, 265-292.

Phillips, J. 1855. Manual of Geology: Practical and Theoretical. London, Charles Griffin and Company, 669 p.

Phillips, T.M. \& DiMichele, W.A. 1992. Comparative ecology and life-history biology of arborescent lycopsids in Late Carboniferous swamps of Euramerica. Annals Missouri Botanical Garden, 79, 560-588.

Potonié, R. \& Kremp, G. 1954. Die Gattungen der Palaőzoischen Sporae dispersae und ihre Stratigraphie. Geologische Jahrbuch, 69, 111-193.

Potonié, R. \& Kremp, G. 1955. Die Sporae Dispersae des Ruhrkarbons, ihre Morphographie und Stratigraphie mit Ausblicken auf Arten anderer Gebiete und Zeitabschnitte. Teil I. Palaeontographica, Abt. B, 98, 1-136.

Pšenička, J. \& Opluštil, S. 2011. Fossil flora from the Újezd u Svatého Křiže Coalfield (Bolsovian, Pennsylvanian), Radnice Basin, Czech Republic. Folia, 45, 61-93.

P̌̌enička, J., Mosca, P., Opluštil, S. \& Martinetto, E. 2017. New late Palaeozoic plant remains in the Ligurian Alps (Italy). Bollettino della Società Paleontologica Italiana, 56, 13-33; doi: 10.4435/BSPI.2017.02.

P̌̌enička, J., Opluštil, S., Ronchi, A. \& Šimunek, Z. 2012. Revision of the Pennsylvanian flora from Val Sanagra in western part of the Southern Alps (Italy). Folia, 46, 31-79.

Punt, W., Hoen, P.P., Blackmore, S., Nilsson, S. \& Le Thomas, A. 2007. Glossary of pollen and spore terminology. Review of Palaeobotany and Palynology, 143, 1-81; doi: 10.1016/j.revpalbo.2006.06.008.

Purkyn̆ová, E. 1979. Fytopaleontologie karbonu ve strukturním vrtu Čeladná SV-6 (hornoslezská pánev). [Phytopaleontologie des Karbons in der Strukturbohrung Čeladná SV-6 (Oberschlesisches Becken)]. Časopis Slezského Muzea Opava (A), 28, 187-190 (in Czech with German abstract).
Purkyňová, E. 1986. Floristická zonálnost žacléřského souvrství v důlním poli Dolu Šverma u Žacléře (vnitrosudetská pánev). [Die floristische Zonalität der Žacléř Formation im neuen Kohlenfeld der Zeche Sverma bei Žacléř (Innensudetisches Becken)]. Časopis Slezského Muzea Opava (A), 35, 57-64 (in Czech with German abstract).

Radczenko, G.P. 1956. Palaeontological Data. New families and genera of fossil plants. All Union Scientific and Research, Geological Institute, Ministery of Geology and Preservation of Mineral Resources of SSSR, Palaeontology, New Series, 12, 185-203 (in Russian).

Ramsbottom, W.H.C., Calver, M.A., Eagar, R.M.C., Hodson, F., Holliday, D.W., Stubblefield, C.J. \& Wilson, R.B. 1978. A Correlation of Silesian Rocks in the British Isles. Geological Society of London, Special Report No. 10, 1-81.

Remy, W. \& Remy, R. 1959. Pflanzenfossilien. Ein Führer durch die Flora des limnisch entwickelten Paläozoikums. Akademie Verlag, Berlin, 285 p.

Remy, W. \& Remy, R. 1977. Die Floren des Erdaltertums. Einführung in Morphologie, Anatomie, Geobotanik und Biostratigraphie der Pflanzen des Paläophytikums. Verlag Glückauf GMBH, Essen, 468 p.

Renault, B. 1882. Cours de Botanique Fossile fait au Muséum d'Histoire Naturelle. Lépidodendrées, Sphénophyllées, Astérophyllitées, Annulariées, Calamariées. G. Masson Éditeur, Paris, 194 p.

Renier, A., Stockmans, F., Demanet, F. \& Straelen, V. van 1938. Flore et Faune Houillères de la Belgique. Introduction à l'Étude Paléontologique du Terrain Houiller. Musée Royal d'Histoire Naturelle de Belgique, Bruxelles. Texte: 1-317. Planches: 1-49, pls 1-144.

Rex, G.M. \& Chaloner, W.G. 1983. The experimental formation of plant compression fossils. Palaeontology, 26, 231-252.

Roehl, D. von 1868. Fossile Flora der Steinkohlen-Formation Westphalens einschliesslich Piesberg bei Osnabrück. Palaeontographica, XVIII (4-6), 1-192.

Sauveur, M. 1848. Végétaux fossiles des terrains houillers de la Belgique. Académie Royale des Sciences, des Lettres et des Beaux-Arts de Bélgique, pls I-LXIX.

Schimper, W.Ph. 1869-1874. Traité de Paléontologie Végétale ou la Flore du Monde Primititf dans ses Rapports avec les Formations Géologiques et la Flore du Monde Actuel. Texte - I (1869): I-IV, 1-738; II (1870-72): 1-966; III (1874): 1-896. Atlas (1874): pls I-CX. J.B. Baillière et Fils, Paris.

Scott, D.H. 1909. Studies in Fossil Botany. Second Edition. A \& C Black, London, 683 p.

Smith, A.H.V. \& Butterworth, M.A. 1967. Miospores in the coal seams of the Carboniferous of Great Britain. Special Papers in Palaeontology, 1, 1-324.

Spiekermann, R., Uhl, D., Wanderley Benício, J.R., Guerra-Sommer, M. \& Jasper, A. 2018. A remarkable mass-assemblage of lycopsid remains from the Rio Bonito Formation, lower Permian of the Paraná Basin, Rio Grande do Sul, Brazil. Palaeobiodiversity and 
Palaeoenvironments, 1-16; doi: 10.1007/s12549-0180318-3.

Sternberg, K. von 1820-1838. Versuch einer geognostisch-botanischer Darstellung der Flora der Vorwelt. I (1820-1825) - 1 (1820): 1-24, Tafn I-XIII; 2 (1821): 1-33, Tafn XIV-XXVI; 3 (1823): 1-40, Tafn XXVII-XXXIX; 4 (1825): 1-48, Tafn XL-LIX, Tafn A-E. II (1833-1838) - 5/6 (1833): 1-80, Tafn I-XXVI; 7/8 (1838, Presl): 81-220, Tafn I-LXVIII, Tafn A-B, (incl. Corda: Skizzen zur vergleichenden Phytotomie vor- und jetztweltlicher Pflanzenstämme, p. I-LXXI).

Stockmans, F. \& Willière, Y. 1952. Quelques végétaux namuriens de la Galerie de Ben. In: Étude géologique du Gisement Houiller d'Andenne-Huy (van Leckwyck, W., Demanet, F., Willière, Y \& Chaudoir, H.). Publications Association pour l'Étude de la Paléontologie et de la Stratigraphie Houillères, Bruxelles, 11, pls A-F.

Stockmans, F. \& Willière, Y. 1952-1953. Végétaux namuriens de la Belgique. Association pour l'Étude de la Paléontologie et de la Stratigraphie houillères, 13. Texte (1953): 1-382. Atlas (1952): 1-12, pls I-LVII.

Šusta, V. 1928. Stratigraphie des Ostrau-Karviner Steinkohlenreviers im Lichte der Paläontologie. In: Der Kohlenbergbau des Ostrau-Karviner Steinkohlenreviers (Direcktoren-Konferenz des Ostrau-Karviner Steinkohlenreviers in Mährisch Ostrau im Zehnten Jahre der Čechoslovakischen Republik), 381-484.

Tenchov, Y.G. 1987. Les fossiles de Bulgarie. I.1. Paléozoïque. Flore fossile. Megaflore. 1. Sphenopsida et Lycopsida. Editions de l'Academie Bulgare des Sciences, 1-165.

Thomas, B.A. 1967. Ulodendron: Lindley and Hutton and its Cuticle. Annals of Botany, N.S., 31 (124), 775-782; doi: 10.1093/oxfordjournals.aob.a084181.

Thomas, B.A. 1968. The carboniferous fossil lycopod Ulodendron landsburgii (Kidston) comb. nov. Journal of natural History, 2, 425-428; doi: 10.1080/00222936800770401.

Thomas, B.A. 1986. In search of fossil plants: the life and work of David Davies (Gilfach Goch). Amgueddfa, Bulletin National Museum of Wales, Geological Series, 8, 1-54.

Thomas, B.A. \& Brack-Hanes, S.D. 1984. A new approach to family groupings in the lycophytes. Taxon, 33, 147-255; doi: $10.2307 / 1221165$.

Thomas, B.A. \& Purdy, H.M. 1982. Additional fossil plants from the Drybrook Sandstone, Forest of Dean, Gloucestershire. Bulletin British natural History (Geology), 36, 131-142.

Thomas, B.A. \& Seyfullah, L.J. 2015. A new look at Lepidodendron ophiurus Brongniart and the recognition of L. wingfieldense sp. nov. (Flemingitaceae, Lepidodendrales) from the Langsettian (Bashkirian) of Derbyshire, East Midlands, UK. Palaeontographica, Abt. B, 292, 23-32.

Thomas, B.A. \& Spicer, R.A. 1986. Meyenodendron borealis gen. et sp. nov., a new lepidodendrid of angaran affinity from northern Alaska. Bulletin British natural History (Geology), 40, 293-297.

Veldt, van der 2008. Fossielen uit het Boven-Carboon (Westfalien-A) van Zuid-Limburg, collectie-Gerard van der Veldt. Gea, Stichting Geologische Aktiviteiten, 41, 61-93.
Wagner, R.H. 1959. Flora fósil y estratigrafía del Carbonífero de España NW. y Portugal N. Estudios Geológicos, XV, 393-420.

Wagner, R.H. 1960. Middle Westphalian floras from northern Palencia (Spain) (in relation with the Curavacas phase of folding). Estudios Geológicos, XVI, 55-92.

Wagner, R.H. 1965. Palaeobotanical Dating of Upper Carboniferous Folding Phases in NW. Spain. Memorias del Instituto Geológico y Minero de España, LXVI, 1-169.

Wagner, R.H. 1983. The palaeogeographical and age relationships of the Portuguese Carboniferous floras with those of other parts of the western Iberian Peninsula. In: The Carboniferous of Portugal (eds. Lemos de Sousa, M.J. \& Oliveira, J.T.). Memórias dos Serviços Geológicos de Portugal, 29, 153-177.

Wagner, R.H. 1999. Peñarroya, a strike-slip controlled basin of early Westphalian age in Southwest Spain. Věstnik Českého geologického ústavu, 74, 3-23.

Wagner, R.H. 2001. Fósiles vegetales. In: Recursos Naturales de Córdoba, 6, 105 p. Departamento de Medio Ambiente y Protección Civil, Diputación de Córdoba.

Wagner, R.H. 2004. The Iberian Massif: a Carboniferous assembly. Journal of Iberian Geology, 30, 93-108.

Wagner, R.H. 2013. El significado geológico de las cuencas carboníferas con flora fósil en Sierra Morena. In: Libro de resúmenes (eds. Álvarez-Vázquez, C. \& López Rodríguez, I.). XXIX Jornadas de Paleontología, La Paleontología del Paleozoico, Córdoba, 41-56.

Wagner, R.H. \& Álvarez-Vázquez, C. 1995. Upper Namurian/ lower Westphalian of La Camocha, Asturias: Review of floral and faunal data. Coloquios de Paleontología, Universidad Complutense, 47 (volumen homenaje al Prof. B. Meléndez), 151-176.

Wagner, R.H. \& Álvarez-Vázquez, C. 2008. A revision of the lower Pennsylvanian Alethopteris lonchitica (auctorum) and its identity with Alethopteris urophylla. Revista Española de Paleontología, 23, 157-192.

Wagner, R.H. \& Álvarez-Vázquez, C. 2010. The Carboniferous floras of the Iberian Peninsula: A synthesis with geological connotations. Review of Palaeobotany and Palynology, 162, pp. 238-324; doi: 10.1016/j.revpalbo.2010.06.005.

Wagner, R.H. \& Bowman, M.B.J. 1983. The position of the Bashkirian/Moscovian boundary in West European chronostratigraphy. Newsletters on Stratigraphy, 12, 132161; doi: 10.1127/nos/12/1983/132.

Wagner, R.H., Carballeira, J., Ambrose, T. \& Martínez García, E. 1984. Mapa Geológico de España E. 1:50.000, Hoja ${ }^{\circ}$ 107 (Barruelo de Santullán). Memoria explicativa, 113 p. Instituto Geológico y Minero de España, Madrid.

Wagner, R.H., Coquel, R., Gabaldón, V., Robledo, J., Quesada, C., Hernández, J. \& Rodríguez, P. 1983. The Westphalian B of the Peñarroya-Belmez-Espiel Coalfield. In: Guidebook Field Trip D, Carboniferous Geology of the Sierra Morena (coord. Quesada, C. \& Garrote Ruíz, A.). Dixième Congrès International de Stratigraphie et de Géologie du Carbonifère, Madrid 1983, 53-64.

Witham, H.T.M. 1833. The Internal Structure of Fossil Vegetables found in the Carboniferous and Oolitic deposits 
of Great Britain, Described and Illustrated. Adam \& Charles Black, Edinburgh, 84 p.

White, D. 1899. Fossil flora of the lower coal measures of Missouri. United States Geological Survey, Monographs, XXXVII, 1-467.

Wood, H.C. 1860. Contributions to the Carboniferous Flora of the United States. Proceedings of the Academy of Natural Sciences of Philadelphia, XII, 236-240.

Zalessky, M.D. 1904. Végétaux fossiles du terrain carbonifère du bassin du Donetz. I. Lycopodiales. Mémoires du Comité Géologique, Nouvelle série, 13, 1-126.

Zalessky, M.D. 1907. Beiträge zur Kenntnis der fossilen Flora des Steinkohlenreviers von Dombrowa. Mémoires $d u$
Comité Géologique, Nouvelle série, 33, 1-69 (in Russian with German summary).

Zalessky, M.D. 1934. Carboniferous flora of North Caucasus. The Central Scientific Geological and Research Institute, Geological Survey Section, 1-22 (in Russian with English summary).

Zeiller, R. 1878-1879. Végétaux Fossiles du Terrain Houiller. Explication de la Carte Géologique de la France. IV (2). Texte (1879): 1-185. Atlas (1878): pls CLIX-CLXXVI (offprint 1880).

Zeiller, R. 1886-1888. Bassin Houiller de Valenciennes. Description de la flore fossile. Études des Gîtes Minéraux de la France. Texte (1888): 1-729. Atlas (1886): pls I-XCIV. 
